Florida International University FIU Digital Commons

\title{
An Analysis of Haitian Parents' Perceptions of Their Children With Disabilities
}

Josee Gregoire

Florida International University, jgregoire@dadeschools.net

DOI: $10.25148 /$ etd.FI10041611

Follow this and additional works at: https://digitalcommons.fiu.edu/etd

\section{Recommended Citation}

Gregoire, Josee, "An Analysis of Haitian Parents' Perceptions of Their Children With Disabilities" (2010). FIU Electronic Theses and Dissertations. 152.

https://digitalcommons.fiu.edu/etd/152

This work is brought to you for free and open access by the University Graduate School at FIU Digital Commons. It has been accepted for inclusion in FIU Electronic Theses and Dissertations by an authorized administrator of FIU Digital Commons. For more information, please contact dcc@fiu.edu. 
FLORIDA INTERNATIONAL UNIVERSITY

Miami, Florida

\section{AN ANALYSIS OF HAITIAN PARENTS' PERCEPTIONS OF THEIR CHILDREN WITH DISABILITIES}

A dissertation submitted in partial fulfillment of the requirements for the degree of DOCTOR OF EDUCATION

in

EXCEPTIONAL STUDENT EDUCATION

by

Josee Gregoire 
To: Interim Dean Marie McDemmond

College of Education

This dissertation, written by Josee Gregoire, and entitled An Analysis of Haitian Parents' Perceptions of their Children with Disabilities, having been approved in respect to style and intellectual content, is referred to you for judgment.

We have read this dissertation and recommend that it be approved.

$\begin{array}{r}\hline \text { Delia Garcia } \\ \hline \text { Joyce Harth } \\ \hline \text { Diana Valle-Riestra } \\ \hline \text { Maria Elena Villar } \\ \hline \text { Elizabeth Cramer, Major Professor }\end{array}$

Date of Defense: March 23, 2010

The dissertation of Josee Gregoire is approved.

Interim Dean Marie McDemmond

College of Education

Interim Dean Kevin O'Shea

University Graduate School

Florida International University, 2010 
(C) Copyright 2010 by Josee Gregoire All rights reserved. 


\section{DEDICATION}

I dedicate this dissertation to my mother Anne Enise (deceased) and my father Louis Volvic. They instilled in me many core values and beliefs. They thought me the value of hard work, commitment, courage, strength, love of family, the value of knowledge, the importance of having dreams and aspirations and, most of all, a strong sense of identity. My mother, especially, faced terminal illness, and ultimately, death with extra-ordinary courage and strength even to the end. In doing so, she taught me to value life, to have a vision and to be goal-oriented. The completion of this degree meant so much to me because she never had the opportunities that I have been given. I would not be who I am today without the values and beliefs my parents instilled in me. 


\section{ACKNOWLEDGMENTS}

I wish to express my deepest gratitude to Dr. Elizabeth Cramer for her willingness to take on the enormous task of chairing my committee so late into the process of finishing this degree. She provided guidance, support and assistance under enormous time pressures. I am eternally grateful to her for her dedication to this project.

I would also like to thank Dr. Patricia Barbetta for the contribution of her time, knowledge, and expertise during topic selection and development. Her assistance was paramount in sustaining my interest in this topic.

My heartfelt appreciation goes to the members of my committee, Dr. Delia Garcia, Dr. Joyce Harth, Dr. Diana Valle-Riestra, and Dr. Maria Elena Villar. Their help and support was invaluable to me.

Dr. Luretha Lucky also deserves to be recognized for her help and support during my earlier years at FIU. She motivated me to get started on this path and was instrumental in helping me to shape and mold my research and presentation skills.

My family has been a constant source of help and motivation for me to stay the course and see this project through. I would especially like to thank my sister and best friend, Mariline. She has always been there for me. I also acknowledge my other siblings, brothers-in- law, and numerous nephews and nieces. They served as reminders to me, to not to give up because they always believed in me.

Finally, I have had a network of friends and advisors who over the years always encouraged and believed in me. This degree is a testament to what they believed I could accomplish even when I doubted myself. I am grateful for their faith in me. 


\section{ABSTRACT OF THE DISSERTATION \\ AN ANALYSIS OF HAITIAN PARENTS' PERCEPTIONS OF \\ THEIR CHILDREN WITH DISABILITIES}

by

Josee Gregoire

Florida International University, 2010

Miami, Florida

Professor Elizabeth Cramer, Major Professor

Parental involvement is an integral part of the educational system in the U.S. Yet, parents from non-mainstream racial/ethnic backgrounds have not fully grasped the nature of parental involvement expectations in the educational process and how these expectations may impact student achievement. The purpose of this study was to identify Haitian parents' perceptions of their children with disabilities and the education these children were receiving.

Several authors have conducted studies on parents of children with disabilities to better gain an understanding of the level of their involvement with their children's education, their perceptions of the children, and their views on the school system (Harry, 1992a, 1992b).

In this study, Haitian parents of children with disabilities were interviewed using an interview protocol. Through these interviews, this study explored 10 Haitian parents' perceptions of their child with a disability, the education the child was receiving, their interaction with the school system, and how the disability had affected their relationship with their child and their involvement with the school. 
Findings of the present study revealed that these Haitian parents seldom disagreed with school personnel and did not seem to fully grasp the different methods available to address their concerns as parents of children with disabilities nor the role they were expected to play in the process. The majority did not have basic literacy skills in Creole or English. The parents in this study were overwhelmed by school written communication.

Additionally, this study discovered that parents' perceptions were guided by two core concepts: coping mechanisms and locus of control. Parents with an internal locus of control, who tended to be more educated, focused inward to find solutions to problems encountered. Those with an external locus of control relied on outside influences to resolve their problems.

Parental involvement was strongly influenced by their values, beliefs, customs, and conceptual knowledge about disability; all closely aligned with culture and acculturation. Overall, these parents' perceptions greatly influenced their thoughts and behaviors when they realized that their children with disabilities might fall short of their immigrant dreams of success they held for these children. 
$\begin{array}{ll}\text { CHAPTER PAGE } & \text { PAT }\end{array}$

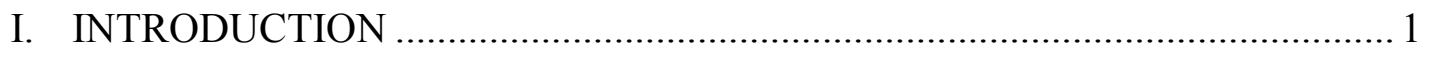

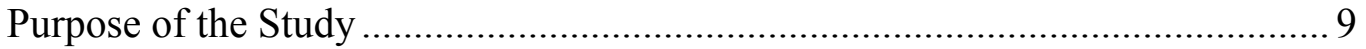

Statement of the Problem................................................................................ 10

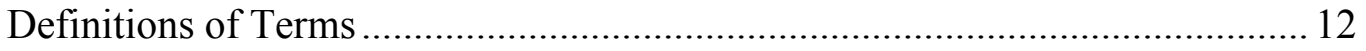

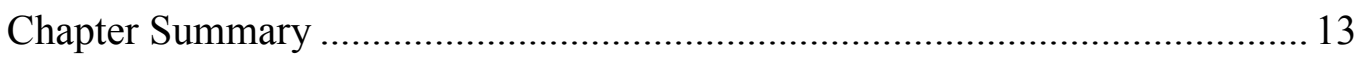

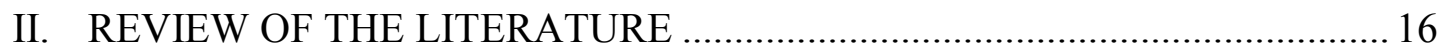

Parental Involvement in General Education .................................................... 16

Parental Involvement Within Minority and Culturally and

Linguistically Diverse Communities ............................................................. 27

Parental Involvement With Minority, CLD Children, and

Children With Disabilities ............................................................................... 36

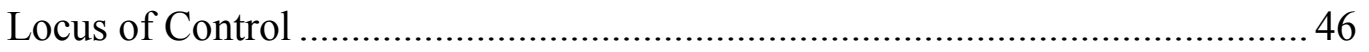

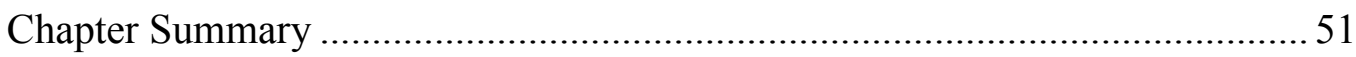

III. METHODS …....................................................................................... 53

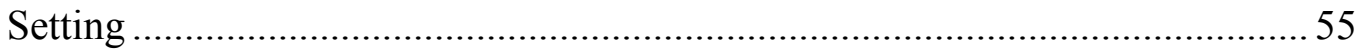

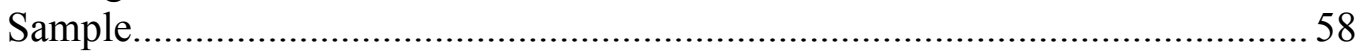

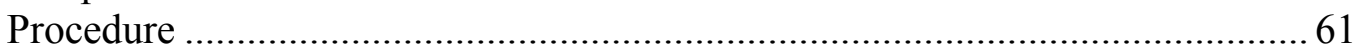

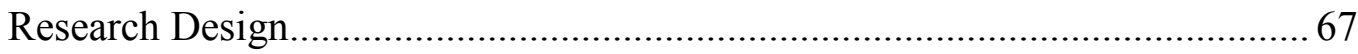

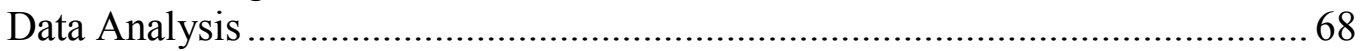

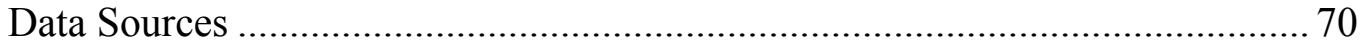

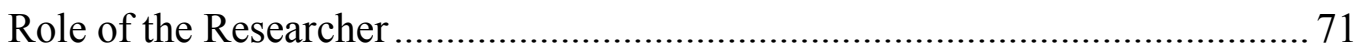

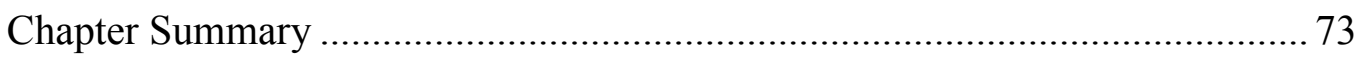

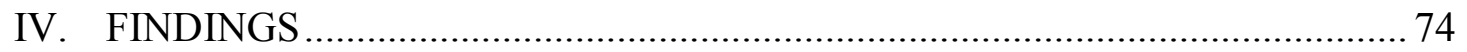

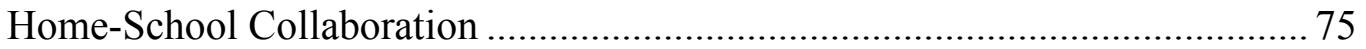

Parental Concerns .................................................................................. 85

Relationships/Perceptions ..................................................................... 91

Core Concepts: Coping Mechanisms and Locus of Control............................. 98

Chapter Summary …........................................................................ 103

V. DISCUSSION AND CONCLUSIONS .................................................... 106

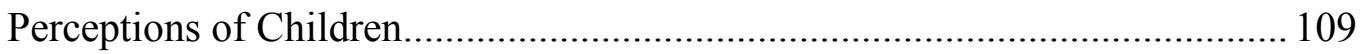

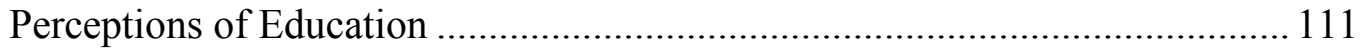

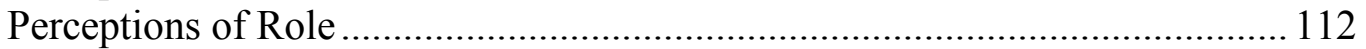

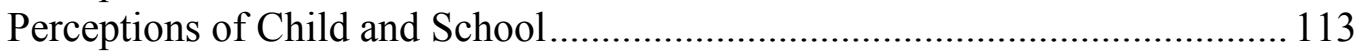

Perceptions of Support/Barriers .............................................................. 115

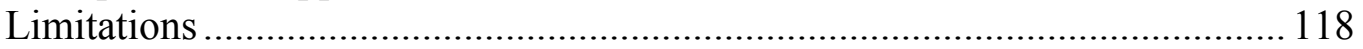


Implications for Current Practice .......................................................... 120

Recommendations for Future Research ....................................................... 125

Discussion Summary .............................................................................. 128

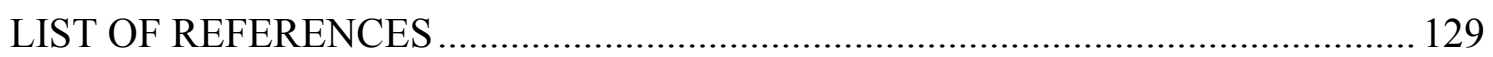

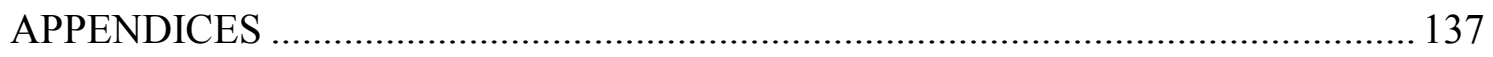

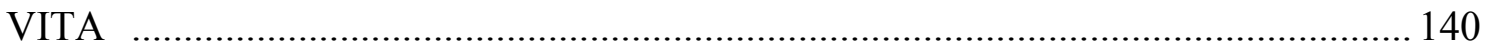




\section{CHAPTER I}

\section{INTRODUCTION}

Haiti was the first nation in the western hemisphere to successfully win independence from colonial occupation. Prior to independence in 1804, Haiti was a French colony. Following the successful revolt of the slaves against the French, Haiti became isolated from the rest of the world. For the next 150 years or so, France's educational system, its customs, culture and mores permeated Haiti. Children of Haiti's middle and upper classes tended to immigrate to France for advanced studies (Stepick, 1998). Furthermore, the historically drastic shifts in political power in Haiti's governments since independence have led to the exile of the majority of its past leaders and they have tended to emigrate to French speaking countries in Africa or Europe (Stepick, 1998).

Haiti's tumultuous political transitions have resulted in economic and social impacts for the country's population. The last political impact that significantly changed the societal make-up of the country started following the presidency of Francois "Papa Doc" Duvalier in 1957. Duvalier established a dictatorship that instituted severely repressive measures and harsh punishment for those whose ideals or visions were not in accordance with his philosophy. The repressive political atmosphere led to the exodus of the upper middle class around 1964 as a reaction to the brutal repression and lack of economic security (Stepick, 1982). This first wave emigrated to France, Canada, Africa and the United States (U.S.), and more precisely, New York City. As the political and economic situation worsened in the Haiti, the middle and lower classes also began to leave the 
country in mass in the second wave. In the seventies they started to choose South Florida as one of their destinations. This influx of Haitians continued until 1980 when it reached a climax with an exodus of Haitians arriving by boat on the shores of Miami until U.S. Immigration policies began to repatriate them. The last wave arriving by boat was of a lower economic and educational level than their previous countrymen. The 1980s and thereafter saw a burgeoning Haitian population settling in Miami changing the landscape of the South Florida community forever. Most settled in Miami-Dade initially, but many have settled in other parts of the state, most notably Broward County and further north to a lesser degree (Stepick \& Stepick, 2003). Haitians have come to the U. S. because of the promise of better economic and educational opportunities for themselves and their children as well as greater political freedom and to escape persecution. From the U.S., they have kept themselves abreast of the economic and political situation in their homeland while simultaneously taking steps to adapt themselves to life here in the U.S.

Most of the Haitian refugees arriving by boat in the 1980s on the shores of South Florida were relatively young and women outnumbered the men in a study conducted by Stepick (1998). He found that almost 50\% of the participants in the research were single. However, in a follow-up study done with the same sample, the researcher found an overwhelming increase in marriages and children. Sixty-one percent of those participating in the follow-up had at least one additional child from the first contact to the follow-up 2 years later (Stepick, 1998). Stepick, (1998) also found that these participants had moved at least once during the span of time between the first and second contact. Approximately one fourth had moved twice. The number of those owning their homes as opposed to renters also had doubled in the 2 year span. The two factors that remained 
constant had to do with social integration. The first was socialization with other racial/ethnic groups and neighborhood of residence, which remained the same. The participants had not formed friendships outside their racial/ethnic group and most had stayed in the same vicinity. The second was mobility. Almost two thirds continued to live in the same predominantly Haitian neighborhoods where they had previously resided. As a result, or perhaps, as a by-product, these Haitians also tended to socialize mostly with others from the same racial/ethnic group. More than $75 \%$ had no Anglo or African American friends. More than $90 \%$ socialized mostly with other Haitians. They worked in predominantly low-income jobs such as maids, janitors, kitchen helpers and other similar jobs. Stepick (1998) found, however, that this group of immigrants exhibited an ambition and drive to excel. This was demonstrated by the fact that although as a group only $5 \%$ had graduated high school prior to leaving Haiti, more than $50 \%$ enrolled in school within the first 3 years of living in South Florida. The major difference between this group of immigrants who arrived in the early 1980s and others who had arrived prior to that time is that this group of immigrants had less education, but they were certainly more educated than the ones who still remained behind in Haiti. For the most part, Haitians perceived school as the vehicle through which one could rise above current circumstances (Stepick, 1998). Therefore, although many had started in school by taking English classes, 2 years later, they had progressed to vocational, high school and college classes.

As Haitians integrated into the fabric of South Florida by obtaining better jobs, home ownership and families, their children were also entering the public school system. Stepick's research (1998) and that of others have found that Haitians like other immigrant groups usually exhibited high academic potentials, but that this potential did not 
necessarily lead to a positive outcome. Conflicts arose between the parents' perception of the American educational system and their children's behavior. As their children strove to imitate and fit into the culture and norms of their adopted county, parents perceived it as a rejection of themselves and the values they had tried to instill in the children (Stepick and Stepick, 2003). These conflicts within the family oftentimes lead to discord.

The findings of a longitudinal project conducted by Stepick and Stepick (2003) presented the characteristics of immigrant children and how their interaction with the environment influenced their social values and ultimately their educational outcomes. Because immigrant children were perceived as having different behaviors and appearance, many felt ostracized and felt the pressure to assimilate so that they could blend more easily into the mainstream culture. However, this change in appearance and behavior caused friction within the family because the parents perceived it as a rejection of their own values and their children's failure to appreciate the opportunities they had tried to give them by coming to the U.S. (Stepick \& Stepick, 2003). Young adolescent immigrants faced difficulties in trying to maintain their home country's values and beliefs while at the time trying to achieve academic success and assimilation. This was especially true for immigrants of African descent because of the differences between their home county and that of African Americans (Stepick \& Stepick, 2003).

As children assimilated into the American educational system, they were more likely to reject their parents' cultural values as a norm, especially during the early high school years when fitting in was at the forefront of their minds. Parents' cultural values and beliefs took a back seat to peer pressure from the dominant culture. Students were often 
at odds with their parents during these high school years when they were feeling the pressure to model the look and behavior of the dominant culture and parents were alienated and shunned for their different values and beliefs. At the same time, parents were also reluctant to abandon their children to a culture that was not understood nor trusted. The situation was further exacerbated by the parents' belief that education was their way and their children's way out of poverty and the very reason why they left their homeland (Stepick \& Stepick 2003). Therefore, parents were at an extreme disadvantage when seeking to support their children in the educational process because of a lack of awareness of what their expected roles were in the process, and how to initiate and maintain involvement to the benefit of their children. The role of parents here in the United States is so different from that of parents in Haiti. For example, parents in Haiti left their children at the door and were not expected to maintain ongoing communication with the school. As the number of Haitian students in the public school system increases, it is becoming more vital for Haitian parents to understand the nature of the system, how it works and what their rights and responsibilities are towards their children.

The educational system in America includes parent participation and input in the process of educating children. Research has documented the importance of parental involvement in promoting positive student performance and increasing educational achievement (Chavkin, 2005; Chrispeels \& Gonzalez, 2004; Halsey, 2004; HooverDempsey, Bassler \& Brissie, 1992; Hoover-Dempsey, Bassler, \& Burrow, 1995; HooverDempsey, Walker, Jones, \& Reed, 2002; Musti-Rao \& Cartledge, 2004). Parental involvement serves an important role in the educational process and has a direct impact on both student achievement and school success (Sensiper, 1999). Parental involvement 
has been shown to influence motivation to learn, improve consistent attendance patterns, and decrease dropout rates and behavior problems (Chispeels \& Gonzalez, 2004; Ginsberg \& Herman-Ginsberg, 2005). In the American educational system, parental involvement is an integral part of the educational process, and there are many factors that can influence that involvement. However, this involvement can be problematic for parents who are not familiar with the American educational system (Ariza, 2002; Harry 1992a; Wherry, 2007b). Therefore, it remains important to explore not only the factors that contribute to parental involvement, but also the rationale behind different levels of involvement among different groups of parents.

According to the U.S. census statistics (2000), the demographics of the country have been changing over the past 20 years. Data quoted have identified several shifts in populations to underscore this phenomenon. The general population grew from 248.8 million in 1990 to 272.7 million in 1999, (U.S. Census, 2000). This data reflected subtle changes in the country's ethnic make-up. Data from a different source was disaggregated (Schmidley, \& Robinson, 2003; Smith, Ahmed, \& Sink, 2000). The numbers from these other sources indicated that the Anglo population grew by $7.6 \%$ to 224.6 million; the African American population by $14.4 \%$ to 34.9 million; the Asian and Pacific Islander populaton by $44 \%$ to 10.8 million; the American Indian and Alaska Native population by $14.3 \%$ to 2.4 million; and the Hispanic population by $39.7 \%$ to 31.3 million people. The Anglo population had the smallest increase when compared to all the other racial/ethnic groups identified in the data. When analyzed by region, the data indicated different proportions across regions of the U.S. (Schmidley, \& Robinson, 2003; Smith, Ahmed, \& Sink, 2000). 
There were three racial/ethnic groups that demonstrated noticeable shifts in mobility among the regions of the U.S. African Americans, Asian and Pacific Islanders, and Hispanics had the largest increases in the Southern and Western regions of the U.S. with the most populous states being Arizona, California, Texas and Florida. These shifts have made the U.S. more culturally and linguistically diverse (CLD) and added new dynamics to the demographic landscape of the country. The changes were also reflected in the school age population that emerged in this country based on data gathered between 1990 and 2000 (Johnson, Kominski, Smith, \& Tillman, 2005). The changing demographics in the U.S. have resulted in more children born of immigrant parents residing here. These children come to school with different customs, values and beliefs impressed upon them by their parents. Moreover, these parents have not internalized the U.S. educational expectation of parental involvement in schools (Ariza, 2002). Although the combined percentage of children living in the U.S. exhibited minute fluctuations from $25.4 \%$ of the population to $25.5 \%$, the diversity among these children was clearly evident. Children of Hispanic heritage grew by $5 \%$ from 12 to $17 \%$. The percentage of children who spoke a second language at home also rose by the same percentage within the same time period. These numbers reflected a change in cultural and linguistic diversity. They also demonstrated a higher percentage of children coming from homes where parents were not fluent in the English language and parents who spoke a second language at home. The U.S. Census Data (2000) revealed that the number of children with at least one parent from a foreign country grew by a 40\% margin between 1990 and 2000 , another indicator of the increase in diversity in this country. 
The number of Haitian parents with school age children has been on the rise in this country in general, and in South Florida specifically, for a number of years (Stepick, 1998). Haitians are a sub-group of the African American population that is not specifically identified as a separate ethnic group. In all data collected by ethnicity, African Americans or Blacks are considered one group, regardless of country of origin. Based on Census data from 2000 (Zephir, 2004), the number of Haitian immigrants in the U.S. was estimated to be approximately 385,000 . However, analysts from Miami-Dade County, New York City and Homeland Security estimated these numbers to be closer to 1 million in actuality (Zephir, 2004). Therefore, in order to identify any sub-group of African American or Black ethnicity, it is necessary to extrapolate the numbers from the data available to arrive at a reasonable number to form estimates.

Acculturation and assimilation are two ways for the foreign born to blend more easily into the American society, and they are facilitated by English language proficiency. Parents who were more proficient in the English language exhibited greater communication skills. These parents, in turn, demonstrated greater feasibility to participate in children's education and to familiarize themselves with U.S. norms and customs (Al-Hassan \& Gardner, 2002). The data provided by Johnson, Kominski, Smith and Tillman (2005) revealed that $75 \%$ of foreign born parents who had children reported that they had difficulty communicating effectively in the English language. This number represented a $21 \%$ increase from the previous decade. The children of these parents, therefore, spoke a foreign language more frequently at home because their parents were less proficient in English. As a result, these children tended to develop fluency in both English and a second language in order to communicate with their parents while the 
parents attempted to acquire the English language (Johnson, Kominski, Smith, \& Tillman, 2005).

\section{Purpose of the Study}

The purpose of this study was twofold. Parental involvement is an integral part of the educational system in the U.S. Yet, the changing demographics of our county have changed the educational landscape due to the increased diversity of students in the educational system. Many parents, especially those from diverse racial/ethnic backgrounds, have not fully grasped the expected nature of parental involvement in the educational process, and its ability to influence student achievement, nor do they understand their expected role in the process. Therefore, it was necessary to examine the perceptions and motivations of parents with the additional layers of being from a diverse racial/ethnic group and having a child with disabilities. First, in order for teachers to influence parental participation and improve the parent-teacher interactions, teachers need to have a working understanding of parents' perceptions of their roles in the process and how those perceptions could influence participation (McWayne \& Owsianik, 2004; Ouimette, Feldman, \& Tung, 2004). Consequently, additional knowledge is needed by teachers and the educational system with regard to parental perceptions. Haitian parents as a sub-group of African Americans are increasingly present in the educational system in south Florida (U.S. Census Bureau, 2009). Research was needed to better identify the nature of their involvement in their children's educational progress and their perceptions of their role.

The second purpose for the study was to address the concept that parental culture and acculturation have been shown to strongly influence parental views of the education 
of their children with disabilities' and their perceptions of their own involvement in the special educational process (Harry, 2001). This study explored the perceptions and expectations of Haitian parents with regard to the education of their children with disabilities. Its focus was the perceptions of Haitian-Creole parents of children with disabilities, one of many growing minority populations in the U.S. and, specifically, south Florida, where the study was conducted. This study sought to specifically probe Haitian parents' perceptions of their children with disabilities and ultimately their involvement in the educational process for these children. Specifically, the research question and sub-questions for this study were as follows:

What are Haitian parents' perceptions of their children with disabilities?

Research sub-questions:

1. What are Haitian parents' perceptions of the education their children are receiving?

2. How do Haitian parents perceive their role in the education of their children with disabilities?

3. How do Haitian parents' perceptions of their child's disability influence their relationship with their child and their involvement with the school?

4. What do Haitian parents see as supports and barriers to their involvement in their child's education?

\section{Statement of the Problem}

Although, there has been a considerable increase in cultural and linguistic diversity in today's schools, most of the research on parental involvement has been conducted with traditional learners from non-minority backgrounds (Halsey, 2004). 
There is much more to be learned about how parents of CLD children view their roles in their children's education and the strategies that foster improved collaboration between the home and school. Additionally, the field of parental involvement within immigrant families with disabled children has even more limited information available. Hence, the goal of this research was to add to the body of knowledge with respect to a specific group of CLD parents who have children with disabilities; that is, Haitian parents in South Florida.

Research suggested that CLD parents may have different perceptions of their roles in the education of their children when compared to the school's parental expectations (Chen, 2005; Diamond, Wang, \& Gomez, 2004; Kato-Otani, 2004). Further, the results of many of these studies have provided guidance as to what approaches educators should take to improve the quality of parental involvement in schools. However, much of this research has limited utility to educators in many of today's schools, particularly in diverse, urban schools. Today's classrooms are more culturally diverse than ever and recent research is suggesting that parents' cultural backgrounds exert a strong influence on how they viewed their role in the education of their children and that different approaches may need to be taken to foster their involvement (Chen, 2005; Chrispeels \& Gonzalez, 2004; Diamond et al., 2004; Griego Jones, 2002; Hurtig, 2004; Kato-Otani, 2004; McWayne \& Owsianik, 2004).

Haitian parents were found to be at an extreme disadvantage when seeking to support their children in the educational process because of a lack of awareness of what their expected roles were and how to initiate and maintain involvement to the benefit of their children (Stepick \& Stepick, 2003). With regard to the education of children with 
disabilities, federal guidelines enacted to protect the rights of the disabled mandated parental involvement in the process (Al-Hassan \& Gardner, 2002; Harry, 2001). Although many parents of children with disabilities initially got involved with the planning of their children's programs in preschool, as children got older parents tended to become disengaged and their involvement in school decreased (Harry, 2002). This change in behavior suggested the possibility of changes in either parents' attitudes towards school or in parents' perceptions of their role in the educational process. The current study sought to pinpoint the rationale behind the shift in behavior. This was important in light of the fact that, for children with disabilities, parental involvement is not only vital to student achievement, but also mandated by federal law. The purpose of the study was to analyze: (a) whether parents understood the role they were expected to play in the process, (b) what perceptions they had of their children and the educational system, and (c) to what extent those perceptions influenced their behavior.

\section{Definitions of Terms}

There are several terms used in the discussion of this study that refer to specific populations or ideas that may not be relevant to other studies. They are listed in alphabetical order.

Acculturation is the rate at which people who were born in a foreign country adapt to and adopt the norms of the mainstream culture. The rate of acculturation can be affected by how much contact one has with people from one's previous culture. The more contact, the slower the rate of acculturation, and vice versa.

Cultural competence is the ability of individuals to identify with people whose culture is different from their own. It usually involves a concerted effort to learn about the 
different customs, values and beliefs held by others that are different from one's own.

Culturally and Linguistically Diverse (CLD) describes parents or students who are from a different culture and/or language than Anglo White Americans.

Immigrants are people who were born in a foreign country, but have now decided to make the U.S. their home for whatever reason.

Mainstream culture is defined as the values, customs and beliefs of Anglo White Americans who are considered to be the mainstream culture. Although they may have ancestors who originated from another culture and language, the current generation is sufficiently removed to be widely accepted as being part of the mainstream culture.

Minority is a term used to identify a racial/ethnic group of individuals whose numbers are considered to be fewer than the mainstream population.

Socialization is to adjust and cooperate with the social norms of a group, usually the mainstream group.

Parental Involvement is the act of engaging in such home activities as socialization with children, assisting with homework completion, or reading with children and/or participating in school activities, that is, volunteering at school, attending parent teacher meetings, participating in school organizations such as the Parent Teacher Association and otherwise maintaining ongoing communication with the school.

\section{Chapter Summary}

This chapter began with a brief history of Haiti and the migration of its people to the U.S. This was followed by a brief history of the assimilation and acculturation of the 
Haitian population, a sub-group of Blacks in a large south Florida urban district. Next, it explored the changing demographics of the U.S. over the later part of the 20th century and how this phenomenon has impacted the general population. It discussed the role that immigration has played in increasing the diversity of the U.S. population, and subsequently the school age population. Next, the issue of parental involvement and its impact on student achievement was presented along with the interaction of race/ethnicity. This topic set the stage for defining how parents from diverse racial/ethnic backgrounds perceived their role in the educational process and how their knowledge base along with their understanding of their roles influenced behavior. This chapter presented a brief overview of the research acknowledging that parents from non-mainstream racial/ethnic backgrounds may have different perceptions than the mainstream culture and, therefore, would display different levels of engagement in their children's education (Hui-Chen \& Mason, 2008). The research questions were embedded in the purpose of the study followed by the statement of the problem. Finally, the chapter ended with a list of definitions of terms that are relevant to this study.

The next chapter reviews the literature by outlining the research conducted thus far in the field of parental involvement. The research findings are divided into three categories: (a) parental involvement within the general education population, (b) parental involvement within minority and CLD communities, and (c) parental involvement within minority, CLD children and children with disabilities. The chapter discusses the research that has been conducted so far with regard to parental involvement within each of these three groups along with the findings. It also reviews the literature on locus of control, a core concept that emerged during analysis of the data. The research questions pertaining 
to the current study are reiterated. Finally, the review of the literature sets the stage for the current research and provides a rationale for how it adds to the body of knowledge currently available regarding parental involvement, minorities and children with disabilities. 


\section{CHAPTER II}

\section{REVIEW OF THE LITERATURE}

The purpose of this research study was to gather information on parental involvement in an effort to determine how it impacts upon student achievement. Parental involvement was defined by Epstein as inter-collaboration between home, school and community with the end result of impacting students' educational outcomes (Nagel and Guest, 2007). Epstein (2008) identified action steps that school can implement to improve parental involvement. Various studies have explored the link between parental involvement and student achievement. This chapter provides a review of the literature on parental involvement with different groups of parents. Presented initially is a review of the research on parental involvement within the mainstream culture. This is followed by the research on parental involvement for minority groups and culturally and linguistically diverse (CLD) populations. Finally, the available research on parental involvement as it relates to children who are CLD and have disabilities is reviewed. This chapter provided a review on locus of control. Although not originally part of the literature review, this section was added because it emerged as a core concept during analysis of the data. This chapter reiterates the research questions and sub-questions and concludes with the purpose for the current study.

\section{Parental Involvement in General Education}

There exists considerable research regarding the effects of parental involvement in the field of general education. Researchers have explored parental involvement in numerous settings to determine the role parental involvement played in student 
achievement and to what extent this involvement influenced student performance. In an effort to identify key aspects of parental involvement that contributed to academic success, Hoover-Dempsey et al. (1992) conducted research on parental views, their involvement in their children's education, and this involvement's impact on student achievement. Questionnaires were completed with parents and teachers. The parent questionnaires focused on parental participation in different activities such as assistance with homework, parent-teacher conferences, telephone calls with teachers and volunteering in the classroom. The teacher questionnaires also included some of the same activities measured in the parent questionnaires.

Findings revealed that parents who had a positive view of their role in their children's education participated more with homework activities and classroom volunteering. They, in turn, spent less time on the phone with teachers. Their children also exhibited higher achievement levels and their children's teachers felt more capable of influencing student achievement because of the higher level of support coming from the parents. The researchers stated that these results had wide ranging implications for parent-school communications.

As an extension of the research within the field of general education, HooverDempsey continued to explore the influence of parental involvement by conducting additional research with other authors. Hoover-Dempsey et al. (1995) conducted research on parental involvement in homework completion. In this research, the researchers chose homework because they determined that it was an activity closely linked to learning where parents, schools, and students each had a role to play. These researchers conducted interviews to identify the strategies used by the parents to achieve homework completion. 
The findings revealed that some of the parents felt ill-equipped to assist their children with homework completion. However, despite some parents' own feelings of inadequacy, an overwhelming majority expressed that it was their duty and responsibility to participate in homework. Hoover-Dempsey et al. (1995) reported that parents felt homework was a normal part of the school experience for their children.

Building on the findings of previous research indicating the positive correlation between parental involvement and student achievement, Hoover-Dempsey and Sandler (1995) conducted research on the premise of previous studies, which had concluded that parental involvement was closely linked to academic success. The researchers intended to delve deeper by seeking to identify specifically why parents became involved in their children's education and how they manifested their involvement. They also sought to measure how the resulting effect of academic success was demonstrated.

Hoover -Dempsey and Sandler (1995) deduced that parental involvement resulted in parents being able to answer three beliefs: (a) parents must have believed that being involved in their children's education encompassed their role as a parent; (b) they must have obtained a certain level of satisfaction from their involvement and felt empowered by it; and (c) they must have become involved because they perceived an invitation or a demand by either their children or the school to become involved. As a result of having internalized these beliefs, parents then engaged in specific behaviors such as instructing children at home and participating in school activities which contributed to academic success.

In a moderate variation of the variable of parental involvement within the general education population, research by Sensiper (1999) applied social marketing techniques to 
education in an attempt to influence parental involvement. Based on the premise that parental involvement was a key component in student achievement, Sensiper convened a meeting of marketing managers. The goal was to formulate strategies that would promote family-school partnerships. The first strategy was for parents to be viewed as potential clients, hence the need to understand their needs, desires, motivations and values. Then, the strategy was to maintain parental involvement through sustained engagement, incentives, encouragement, and rewards. A final strategy was to change parental involvement as a result of the interaction. The author suggested that marketing strategies could be used to effectively influence parental involvement if the applied principles of product, price, place and promotions were used to engage parents in their children's education.

In search of more tangible factors that contributed to parental involvement, the next group of researchers reviewed the content of previous research seeking a rationale for parental involvement and how this involvement was manifested. Hoover-Dempsey et al. (2001) reviewed research on parental involvement in an attempt to determine why parents became involved in their children's education and how this involvement impacted student achievement. Based on the research they reviewed, the authors determined that parental involvement centered on home activities such as homework completion. The studies identified stated that parents felt more empowered to make a difference in this area. By assisting with homework completion, parents were able to positively impact student achievement because they helped children to develop such skills as motivation, self-empowerment and self-control. These skills were found to be conducive to school 
success. Hoover-Dempsey et al. suggested that schools take steps to increase parental involvement in homework completion.

Education is a collaborative effort between home and school. Parents and teachers interact continuously during the educational process. The nature of this interaction has significant impact on student progress. In this next literature review, Griego Jones (2002), analyzed parent perceptions of teacher knowledge to determine how these perceptions influenced parent teacher interaction. In 2002, Griego Jones conducted research to increase pre-service teachers' knowledge of diversity. Prior to this study, the National Center for Education Statistics determined that although over 50\% of teachers were teaching in diverse settings, only one-fifth felt knowledgeable enough to work with the populations they were serving (Griego Jones, 2002). That researcher used parents' perceptions because parents were considered to be a bridge between teachers and students. Parents who participated took part in several focus group interviews with openended questions. Results indicated that parents felt pre-service teachers should learn about the neighborhood, language and culture of the community. The parents also expressed that teachers should be more aware of children's individual differences and personalities. Finally, parents stated that their children were not encouraged to excel and that teachers exhibited low expectations of students in their interactions with parents. Parents overwhelmingly expressed a willingness to participate in their children's education, but it was tempered by lack of knowledge on how to participate, limited time and personal pressures.

In attempting to explore the extent to which home and school communication influenced parental involvement, Caspe (2003), sought to determine the impact that 
teacher perceptions had on parental involvement. That author reported on previous research which had determined that teachers' beliefs about parents influenced how they viewed parents and whether they considered parents to be partners in children's education. Armed with this premise, Caspe (2003) conducted interviews with teachers to ascertain their views on various issues such as home-school communication, roles, and responsibilities. The findings revealed that teachers' perceptions of parental involvement were mostly based on their own observations and communications with those parents. However, teachers' perceptions had been filtered through their own pre-conceived notions regarding the families. Therefore, teachers needed a more comprehensive knowledge base on the parents they worked with prior to engaging them concerning parental involvement.

In addressing yet another aspect of parental involvement, the two researchers in the next article reviewed analyzed the parent-child interaction and its connection to student success in school. Cordry and Wilson (2004) analyzed the amount of time children spent with parents and compared it to the amount of time they spent in school. They provided support as to why parental involvement was vital to reducing the risk factors for academic failure, dropout prevention, increasing positive behavior, and social adjustment. They concluded that parental involvement was closely linked to student success and as parents disengaged, children would become more vulnerable to external influences, which oftentimes tended to be negative.

School activities have provided parents with additional opportunities to be engaged in their children's education and positively impact achievement. In the next research study, Halsey (2004) presented purposeful opportunities for parental 
involvement in school settings and measured the impact of this involvement on student achievement. In 2004 Halsey reviewed a program initiated by two middle school teachers to increase parental involvement in their district. The teachers involved parents by organizing a series of volunteer activities that included an end-of-the-year party and a band trip. The teachers' parental recruitment efforts included letters, phone calls and planned contacts. After securing the commitment of the parents, the teachers provided support, guidance, resources, and close monitoring as the parents planned and executed the events. The parents were given ownership and a sense of responsibility as they planned and completed various prerequisite activities to lead up to the two culminating activities. As a result, Halsey (2004) reported that the parents achieved success and felt empowered. The teachers reportedly concluded that they had a role to play in sustaining parental involvement through ongoing communication with parents.

Over the years, teachers have remained as the primary harbingers of curriculum and instruction with parents playing a supporting role. Curriculum and instruction has benefited from increased parental involvement, by reinforcing the skills in a different setting and providing additional support to teachers. Lunts (2004) used websites geared towards math instruction to engage parents in their children's education. Lunts (2004) analyzed different websites that had been established and rated them based on specific criteria. They were measured based on whether they included general information, content and concepts, contact information, design and maintenance, and security for parents to use. Lunts (2004) concluded that the teachers did not intentionally use the website as a tool to engage parents or the community, although it achieved this end because parents used it to assist their children. The author felt that websites were an 
excellent tool to increase parental involvement and teachers did not use them to the fullest extent possible. The author further concluded that teachers' websites reflected their instructional level, the resources and tools at their disposal, and their skills and knowledge base in the area of parental involvement.

Parents and teachers' perceptions of one another have a tremendous impact on the educational process because these two variables come in frequent contact and both continually influence student behavior. Musti-Rao and Cartledge (2004) conducted interviews with parents and teachers to see what their perceptions were regarding student achievement. The questions were focused around how both parents and teachers described the same problem in an individual child. The researchers also intended to shed light on how parents and teachers would tackle a given problem and whether they would communicate. The results revealed that parents and teachers had consensus on the problems faced by students in school. However, their approaches to solving problems were different and their communications lacked specificity. Parents were given instructions on how to provide assistance at home to help resolve problems. However, the research concluded that the parents' limited background knowledge, lack of time, and limited resources prevented them from implementing those instructions. Musti-Rao and Cartledge (2004) found that teachers, on the other hand, made general comments but did not specify the needs or improvements observed in students.

In seeking to identify specific characteristics of successful parental involvement, these authors conducted research within one school setting. In 2004 Ouimette et al. conducted a case study to identify what made one school successful in engaging the majority of its parents. The researchers stated that their prior experiences with schools 
and parental engagement were varied in the past. Through interviews, the researchers explored such issues as: (a) why parents had chosen this particular school for their children, (b) parental involvement in their children's lives in and out of school, (c) parental expectations regarding their own involvement in their children's education, and (d) whether parents' involvement had changed over time. The findings revealed that the school had made a concerted effort to engage parents from the beginning. There was a purposeful effort on the part of staff to understand parents' cultures, values, prior experiences, dispositions and backgrounds. This knowledge was then used to engage parents both formally and informally. The school had become the catalyst for parental interaction and communication within the broader community.

In 2004, Wherry also attempted to identify the specific characteristics of parental involvement. This research on parental involvement listed the characteristics that contributed to its success. In Wherry's view (2004b) parental involvement was analyzed from the North American perspective. The researcher developed seven components that were determined to be necessary. They included: (a) a measure that both parents and educators value parental involvement, (b) that parents and educators operated from an environment of trust, respect and collaboration, (c) that parents took part in decision making and were viewed as partners in the educational process, (d) that schools created an atmosphere for parents to participate in different ways, (e) that parents were made aware of educators' expectations and vice versa and (f) that schools provided training to increase parents' skills.

In yet another example of the interaction between parents and teachers and how it impacted parental involvement, Ginsberg and Herman-Ginsberg (2005) conducted survey 
research with teachers on parent-teacher interactions. The study compared National Board for Professional Teaching Standard (NBPTS) and non-NBPTS teachers on five factors. The factors were whether: (a) teachers had positive attitudes and practices when working with parents, (b) there were strategies for working with parents who needed more support, (c) teachers made a concerted effort to reach parents, (d) teachers were reaching minority parents, and (e) teachers understood the value of continuous contact with parents. The findings revealed that the NBPTS-certified teachers were more positive than their counterparts in working with parents. They were also more likely to value parental involvement and to view it as a meaningful tool. The NBPTS-certified teachers tended to be more persistent in engaging parents and employed more strategies to achieve this goal.

In a review of research conducted by Jeynes (2005), the author engaged in a metaanalysis of previous research on parental involvement in search of its link to student achievement by reviewing studies. Of the 77 studies reviewed from K-12, almost onehalf were conducted with secondary school students. A little less than $25 \%$ were from elementary schools, and the rest were a mixture of both elementary and secondary students. The results were that parental involvement was related to higher student achievement. The results were consistent regardless of the instrument used to measure achievement. When the researcher delved deeper to identify the method of parental involvement, the strategies used and race/ethnicity were contributing factors. Jeynes (2005) found that the amount of time parents invested was directly related to student achievement. Parental support and expectations also positively impacted student achievement and race/ethnicity did not have as much influence as the other two factors. 
These results provided more data to schools on the role of parents in the educational process.

Wherry (2007) revisited why parental involvement was important and sought to answer the question of whether it continued to be important to student success. He discussed trends in the last 30 years and major reports such as a Nation at Risk published in 1983 and the Coleman Report published in 1966, both of which outlined how critical parental involvement was to student achievement, irrespective of other resources. Wherry stated that the home environment has been and continues to play a major role in students' prior knowledge, attitude, motivation and values, which were all critical to student success, and therefore, educational outcomes. He further stated that the No Child Left Behind Act (NCLB), a national mandate for school reform, continued to recognize the role of parents in the educational process, hence the encouragement for them to get involved. Wherry (2007) stressed the importance of parental involvement in the educational process.

Recently, Ediger (2008) examined the value of parent-teacher conferences and how parental involvement could be mutually beneficial to both parents and teachers. The author dissected the parent-teacher conference and came up with seven reasons why both parties could benefit from the interaction therein. The author identified parameters for such conferences which included: (a) frequency, (b) content to be included and topics to be discussed, and (c) questions to pose. Ediger (2008) also reflected on community values and how parental involvement was part of developing a learning community. This author suggested skills to be developed as part of that learning community should include greater knowledge of school curriculum and specific skills to increase children's 
readiness skills. Ediger (2008) stated student achievement should be an integral part of a learning community. The research on the effects of parental involvement on children's education established a blueprint for collaboration between home and school in various ways to increase student achievement.

The research reviewed so far has clearly established a link between parental involvement and student achievement. Regardless of the method used, the variables at play and the conditions that existed, parental involvement was related to high student performance, parental feelings of efficacy and professional effectiveness. The question is whether the same results were evident when minority groups were examined. Hence, the next section will examine research findings within minority groups to determine whether parental involvement had the same impact with this particular group of parents.

Parental Involvement Within Minority and Culturally and Linguistically Diverse Communities

In 1990, Giles published an article on the on the interaction between Haitian parents and the U.S. educational system. This author explored the issues encountered by these parents and provided interventions that professionals could implement to not only understand these parents from a different perspective, but also provided strategies that can be used by school personnel to intervene with these parents. Giles (1990) presented the case of a 9 year boy who was exhibiting academic difficulties. The author explained the background experiences of the child in Haiti prior to arriving in The U.S. and how these had contributed to the child's current academic problems. The author also discussed the perceptions Haitian parents held of their role in the education of their children. Prior knowledge, the parents own limited educational abilities, lack of language 
proficiency and work schedules were factors that negatively impacted their ability to engage in the behaviors that were expected of them from the American educational perspective. Giles (1990) identified parents' lost of legitimate, expert, referent, reward and coercive power as possible rationale for parents' lack of control over their children. The author stated these challenges had eroded parents' control over their children. In the absence of internal controls traditionally used by these parents to discipline their children, they had relinquished their authority to external forces. The author suggested that professionals work more closely with Haitian parents to ascertain their needs, maintain strong relationships with the parents and provide the supports needed for them to learn about their role in the U.S. educational system.

Parental involvement has been studied by many researchers to determine if the results obtained with the general population were similar to those of racial/ethnic populations and those from culturally and linguistically diverse (CLD) groups. Stepick and Stepick (2003) explored the issue of assimilation. They conducted a longitudinal study on minority groups in Miami, Florida, studying the characteristics of immigrant children and how their interaction with the environment influenced their social values and ultimately, their educational outcomes. Because immigrant children were perceived by peers as having different behaviors and appearance, many felt ostracized by the pressure to assimilate so that they could blend more easily into the mainstream. However, this change in appearance and behavior caused friction within the family because the parents perceived it as a rejection of their own values and their children's failure to appreciate the opportunities they had tried to give them by coming to the U.S. Stepick and Stepick, (2003) concluded that immigrant children's identities did not remain constant. Over time 
children's the racial/ethnic identities changed from identifying with the mainstream culture to identifying with their parents' home culture. However, their outward appearance (i.e., behavior and cultural preferences) continued to mirror that of the mainstream culture. This effect represented a discord between the outer self and inner self. The researchers found that the immigrant students themselves seemed to recognize this discord. Therefore, their relationships with their immigrant parents gave insight as to whether they managed to achieve the academic potential normally identified with immigrant students. The researchers found that first generation immigrants had the highest success rate academically because they were able to balance mastery of the new country's language, customs and behaviors while maintaining their home country's identities and values regarding education and educational success.

Ariza (2000) explored parent-teacher interaction from the American perspective. The researcher presented various examples of teacher-student and parent-teacher interactions that were misinterpreted by teachers because they were analyzed with a mainstream American perspective. Ariza made a case for improving the communication and interaction between parents of minority students and teachers. She suggested that teachers pull back the layers to uncover the underlying causes for parental behaviors, student interaction, and parental involvement rather than jump to conclusions based on the American value systems. Ariza (2000) provided specific activities that teachers could initiate to better engage minority parents and educate them regarding the expectations and components of parental involvement within the American educational system.

To ascertain the influence of communities on schools, Ariza (2002) conducted interviews. Through semi-structured interviews with parents from one minority group, 
the researcher sought to identify ways in which the community could impact school reform, if any, and whether those reforms could have long-term results. The researcher interviewed parents as well as local school officials. Data from archives were also analyzed to corroborate information. This approach was used because Ariza (2002) determined that educators needed to understand how cultural, social, and language differences can serve as barriers that separate schools from the communities they serve. However, students are a product of their communities and school needed to engage parents as members of those communities in order to educate students more effectively. Findings revealed that communities were able to bring reform into the school by forming coalitions, educating themselves regarding their rights and responsibilities and using their newly found voice to influence the school and to pressure school officials to address their needs. In order to do this, parents had to become educated about educational issues and a by-product of this increased knowledge was increased parental involvement in educational matters.

In their research, Chrispeels and Gonzalez (2004) studied Latino parents to determine whether educational programs aimed at increasing parental knowledge would have a positive impact on parental involvement. Parents were surveyed before and after participating in a nine week educational program. Some of the topics covered were: home learning activities, parental attendance at PTA meetings, parental knowledge regarding curriculum standards, and parental expectations for children. Results from the study revealed that there was a strong correlation between parents' knowledge and their involvement. The researchers stated that parenting practices may have exerted a strong influence on parental knowledge and ultimately involvement. 
With a different approach, Hurtig (2004) sought to increase parental involvement. Immigrant parents from poor communities were asked to participate in weekly writing workshops. They were required to come to the school, write narratives regarding their experiences, edit and share selected pieces within the community, both verbally and in print through published articles. The researcher sought to measure what impact the activity would have on the participants as well as their interactions with the larger community.

Results indicated that the activity had a positive impact on the parents in that it increased their self-concepts and self-identities. The parents translated this new selfawareness into their interactions with their children and community. They became empowered and, therefore, integrated themselves more deeply into their children's education. Their skill level also increased over time as they continued engaging in the writing process.

Kato-Otani (2004) measured parental involvement at home with two distinct ethnic groups to determine if their engagement in the same activity would be influenced by cultural beliefs. Japanese and American mothers were observed reading books with their pre-school children at home. Then a questionnaire was used to determine mothers' practices regarding frequency, and what benefits they believed their children gained from the reading activity. The findings indicated that overall, Japanese mothers started reading to their children almost one year earlier. Kato-Otani (2004) found that Japanese mothers tended to read more folklore books while American mothers read more academically oriented books. Japanese mothers also communicated less with their children during the activity while American mothers asked more probing questions of their children. A 
global observation made by the researcher was that Japanese mothers had different expectations of their children and these differences were due to their different cultural approaches to parental involvement and would result in children being prepared for school, but in different ways.

A study on the premise that different minority groups valued different behaviors and attitudes, hence would exhibit different behaviors regarding parental involvement was conducted by Diamond et al. (2004). This research was based on interviews, surveys and observations of African-American and Chinese-American parents to determine how they perceived educational involvement. The results revealed that while AfricanAmerican parents tended to be more involved with home-school interaction, ChineseAmerican parents were more engaged in home-based activities. Both groups of parents relied on extended family and community supports for assistance with child rearing. The researchers found that external supports created a buffer zone within which cultural expectations were reinforced and passed down from one member to another. The researchers concluded that teachers should take into account the influence culture has on parental involvement, recognize the uniqueness of parental involvement as mitigated by each distinct culture and seek ways to engage parents and communities within diverse cultures.

McWayne and Owsianik (2004) conducted a study in an attempt to limit the impact of incidental variables that may have skewed previous findings on parental involvement. In order to isolate the premise that parental involvement has had a positive impact on mixed groups of children, the researchers limited their research to kindergarten students. Ninety-five percent of the participants were low-income African American 
parents. Four percent were Asian American and 1\% was Latino or other. They were from schools within close proximity to each other. The researchers limited the incidental impact of other variables so as to determine the strength of the link between parental involvement and student performance. Findings indicated that children of involved parents exhibited more positive social and behavioral competence. These children also demonstrated higher academic performance and motivation. There was also more interaction between these children and their parents. By contrast, parents who were less involved had children with more behavioral difficulties in school and also reported less contact and more dissatisfaction with the school. These latter parents also identified more personal problems as the reasons why they were not more engaged at school. The authors concluded that the changing demographics of today's society would require schools to increase their efforts to engage parents in non-conventional ways and would also require teachers to move beyond cultural and ethnic stereotypes to involve parents in the educational process in light of the benefits to be gained.

In 2005 Chen conducted research on Chinese adolescents with the assumption that students' academic achievement was influenced by the support they received from parents, teachers, and peers collectively. Based on the findings, Chen deduced that teachers exerted the strongest influence on student achievement. Parents and peers followed, respectively. The researcher pointed out that the results may have been affected by the cultural norms of the society where the study was conducted. Chen (2005) found that female students were more strongly influenced academically by parents and teachers than males. Although both genders were affected by parental support, females showed a 
stronger link between parental support and academic achievement. The researcher stated that this result may have been due to societal expectations regarding gender identity.

Also in 2005, Sheridan, Clarke, Marti, Burt, and Rohlk re-emphasized the value of home-school collaboration to increase student achievement. They reviewed previous research indicating that parental involvement had contributed to increased student attendance, better study habits and a decrease in discipline issues. To add to the body of knowledge, the researchers used a behavioral consultation model to work with parents of young children from diverse backgrounds. First, parental concerns were identified. Next, a plan was developed and school personnel and parents worked towards accomplishing established goals. Finally, student progress towards accomplishment of the goals were measured and modified as necessary. The researchers concluded that purposeful intervention of this type had a positive impact on students behaviorally, socially, and academically. They concluded that home-school collaboration was a key factor in impacting student achievement. The study provided additional evidence of the need for parents and professionals to work together to positively affect student progress.

Cartledge and Kourea (2008) identified characteristics that teachers of children from CLD backgrounds should have so that these teachers could create environments that were more conducive to student achievement. The characteristics were: (a) the ability to assess and provide intervention early to increase readiness skills, (b) a clear understanding of instructional objectives, (c) the ability to provide ongoing assessment that is closely aligned with instruction, and (d) the skill of integrating learning modalities and positive reinforcement in daily instruction. The researchers reviewed various studies that provided support for this theory. These studies demonstrated that student 
achievement matched teacher expectations. Cartledge and Kourea further stated that CLD students and those at risk for having a disability required environments that not only supported them academically but also provided emotional nurturing to ensure success. This emotional nurturing may even extend outside the classroom into social settings within the community with mentors. The researchers made a case for this specialized instruction with documented research findings on CLD and at risk populations and concluded that both schools and individual teachers needed to take an active role in closing the achievement gap for CLD at risk students. This finding provided further justification for home-school collaboration and the need for schools to see parents and communities as partners in the educational process. Cartledge and Kourea (2008) demonstrated that this stance was even more vital when dealing with CLD populations. Klein (2008) explored minority parental involvement and how parents came to terms with educational beliefs and expectations different from their own. The researcher conducted case studies of Chinese-American fathers to determine how they adapted their parental involvement within the American educational system to benefit their children. Klein identified previous research findings on the direct link between parental involvement, school effectiveness and student academic success. The researcher reported that these minority parents developed increased knowledge and awareness of the American educational system through self-initiated interaction with various segments of the community such as other parents, church community events, and probing questions of their children. The parents engaged in this pursuit of knowledge out of a genuine desire to see their children succeed. Klein concluded that the fathers did not acquire the skills needed to become more involved in their children's education through any purposeful 
means initiated by the school. Rather, their children were successful because they actively identified ways to be involved with their children and put it into practice. Therefore, the perceptions of minority parental involvement needed to be fleshed out because parental involvement is so closely tied to student achievement. Greater knowledge and understanding can lead to formulations for increasing parental involvement, especially in minority groups with the end result of positively impacting student achievement.

The literature review on parental involvement within minority groups yielded similar results, although the added challenge was that professionals needed to take more of an active role in involving and including parents. The findings revealed that professionals needed specialized training and sensitivity to work with this population. These parents faced more challenges because they were from diverse backgrounds and therefore, had a different understanding of their role in the educational process. They were also, oftentimes, dealing with their own personal challenges and did not fully grasp what the educational system expected of them. In the next section, the issue of parental involvement is reviewed as well, but with the additional factor of children with disabilities.

Parental Involvement With Minority, CLD Children, and Children With Disabilities

Parental involvement becomes even more critical for parents of children with disabilities. In 1975 Public Law (P.L.) 94-142 (Department of Education) required that parents play a role in the decision-making process for the education of their children. This law reinforced the importance of teachers of students with disabilities to find 
effective ways to work with parents. Since its authorization, this law is periodically reauthorized by Congress and is now widely known as IDEA (Individuals with Disabilities Education Act). With each re-authorization, there have been modifications relating to implementation. However, one aspect of the law that has been constant has been the involvement of parents in the process. Therefore, states and local districts have put into place measures to involve parents in the development of their child's Individual Education Plan (IEP) (Harry, 2002b)

As early as 1992, Harry conducted an ethnographic study of parental involvement with CLD parents of children with disabilities. Through a series of unstructured interviews and observations, the researcher sought to uncover how culture influenced parents' perceptions of their children's with disabilities and how they interacted with school personnel. The findings revealed that parents mistrusted school personnel, felt that the school setting was cold and uncaring towards them, deferred decisions to school personnel even when they did not agree with those decisions, and felt overwhelmed by all the written communication they received regularly from school personnel regarding their children. The findings from this study further illustrated the variances which professionals needed to be aware of when dealing with these parents. Harry (1992a) cited research to support the assertion that CLD parents in many instances were too overwhelmed by personal pressures to fully participate in the educational process, were isolated by limited knowledge and information and exhibited poor self-confidence in their abilities to interact with school personnel. She reported that the general atmosphere of separation and lack of trust was brightened by one recent attempt by the school district to engage CLD parents of children with disabilities through collaboration and respect. 
Also in 1992, Harry reported the findings of a study conducted with CLD parents of children with disabilities on their perceptions of these children and how it affected their parental involvement. Although IDEA mandated parental involvement in special education, the researcher asserted that this involvement would be limited, if professionals could not engage parents meaningfully. Therefore, the current study sought to identify the extent to which culture played a role, if any, in parents' perceptions of their children with disabilities and the level of their engagement with school personnel. It also investigated what factors helped or hindered parental involvement. The findings revealed that culture had a strong influence on parental perceptions because many parents whose children were physically intact and capable of functioning independently within the home and community did not perceive those children as disabled. They agreed with the services provided by school personnel for academic functioning, but not the label. The parents in this study were from non-English speaking countries, making it difficult for them to communicate with school personnel. Their engagement was limited due to their language barriers to a large degree and by cultural differences to a smaller degree.

In 1995, Harry, Allen, and McClaughlin completed a longitudinal study on the engagement of CLD parents of children with disabilities. The researchers reiterated the mandate of parental involvement in the educational process included in IDEA. With that in mind, they conducted interviews and observations of parents of pre-school to elementary children as well as school personnel. They sought to uncover parents' perceptions of their children and whether those perceptions varied over time along with parental participation. The results were that parents initially had a positive perception of their children and the labeling and placement into special education. However, over time, 
this positive perception was reversed and they progressively developed a negative opinion. As a result, their involvement also changed from active participation at meetings to limited or non-existent attendance. The parents were described as being limited in their abilities to participate fully in the process by school personnel. Harry et al. (1995) described a system marred by rigid structures, guidelines and procedures that was inflexible to the needs of CLD parents of children with disabilities and their daily challenges.

In 1999, Harry, Rueda, and Kalyanpur conducted a study to provide insight on how to engage CLD parents of children with disabilities. The researchers based the rationale for their study on the premise that previous research had concentrated on the norms and customs of the mainstream culture and not targeted towards CLD parents who were a key influence on children's behavior and overall development. A longitudinal study was conducted with families over a four year period. In that time, families were observed in their interactions at home, with school personnel and within their communities. Based on the findings, the researchers concluded that professionals working with CLD parents of children with disabilities needed to understand that particular group's ethnic and cultural beliefs, understand that there could be nuances within each particular group and that goals should be framed within each particular families' needs and present levels of development. They suggested that personnel preparation programs needed to prepare individuals to work with these families by ensuring they are flexible, reflective and knowledgeable regarding the current cultural and ethnic beliefs of families. Only then would family involvement be more successful. 
In 2001, Harry was responding to what was perceived as limited knowledge of CLD families in the field of special education when this article was written. The author set the stage by stating that minority groups have not been studied because of their status as a minority group from the mainstream's viewpoint and because in minority groups, the presence of a disability usually took precedence over the cultural and linguistic concerns that may have also been present. She asserted that current trends in the field of CLD with disabilities were forcing researchers to look at culture and disability with social constructs that are influenced by race/ethnicity and rate of acculturation as well as assimilation. Harry concluded that parental involvement, although mandated by IDEA, may also be strongly influenced by values, beliefs, customs and conceptual knowledge that are closely aligned with culture and acculturation. In light of this, Harry (2001) remarked that in order to engage parents more effectively, personnel preparation programs needed to increase the breadth and depth of course content to add culture and its influence on parental involvement.

Palmer, Fuller, Arora, and Nelson (2001) reported on the comments of CLD parents of children with disabilities regarding their support or disapproval of inclusion programs as well as their rationale for their positions on this issue. They conducted a study of CLD parents from different backgrounds who provided feedback regarding inclusionary practices. Parents were asked to respond in writing to a comment by first rating different levels of agreement or disagreement with inclusion and then writing a response. The researchers chose parents of children with moderate to severe disabilities and all children were being served in non-inclusionary settings. Results indicated that while more parents rated inclusionary practices favorably, less supported it for their own 
children in their comments. Many expressed that they felt the inclusion setting was not ideal for their children due to medical, social, self-help, cognitive and motor needs.

Overall, the tendency for parents to prefer one setting over another seemed to be largely dependent upon individual student needs and parents' beliefs. Cultural and ethnic background seemed to be of little, if any significance. This study shed light on the need to take parental perceptions into consideration in parental involvement, especially where children with disabilities were concerned. The next study dealt with parental perceptions and its impact on parental involvement specifically.

Wathum-Ocama and Rose (2002) studied the perceptions, attitudes, and feelings of CLD parents of children with disabilities to determine what impact, if any, these feelings had on parental involvement. Using semi-structured interviews, observations and a review of student records, the researchers employed this method to corroborate data. The criteria used to select participants were that: (a) they lived in the U.S. for more than three years, (b) the parents were not born in the U.S., (c) parents had multiple children including the one with disabilities, and (d) the child with disabilities was enrolled in school. The findings revealed that although the parents indicated interest in participating in their children's education, they lacked specific knowledge on how best to do that. Additionally, their perceptions of these children were that they had lower expectations of them academically because of the disability. Finally, parental involvement was influenced by language barriers and limited knowledge of IDEA and its mandates. Although parents expressed contentment with their children's education, they did not have specific information on how the children were progressing through the educational program. 
In another study, Darch, Miso, and Shippen (2004) presented a model for increasing parental involvement for children with learning and behavioral difficulties. The authors saw increased parental involvement as a precursor to diffusing problems, especially where children with special needs were concerned. In this article and others previously mentioned, Darch et al. (2004) stated that parental involvement led to increased student achievement in school, higher test performance, more consistent attendance, higher rates of homework completion, higher grades, increased motivation and more positive attitudes towards school activities. They identified some of the strategies that could be used to engage parents of these children such as providing behavioral techniques to be used at home with reinforcement, parental support in the classroom and reinforcement of social skills taught at school. According to the authors, parents of children with behavioral difficulties are faced with family stresses such as lack of employment, limited finances and single parent homes. Their model recommended intervention in four phases which encompassed developing materials, recruiting parents applying the strategies to involve parents and providing assistance to sustain engagement.

Murray and Mandell (2004) provided background data to support their claim that families in early childhood special education did not use established family-centered practices effectively. They conducted a study with personnel enrolled in an early childhood special education program to determine their perceptions regarding the use of family centered practices. The study consisted of interviews following participation in a program model designed to increase knowledge of family centered practices. They attributed this to the fact that professionals failed to engage these families. Results indicated that participation in the family centered program increased professionals' 
ability to engage families, improved their attitudes and beliefs regarding families and increased professional confidence and skill in implementing family centered practices. The authors stated that professionals failed to engage parents because they had limited knowledge and understanding of the needs and perceptions of families with disabled children. The authors remarked that although the results supported the value of using professionals to increase parental involvement with specialized populations, caution should be used in over generalizing the outcome because of the sample size and the single method used to measure effectiveness of the model.

Recently, Arzubiaga, Artiles, King, and Harris-Murri (2008) engaged in an examination of the research currently available on CLD families in attempt to determine whether the knowledge gained from the research has been translated into practice. They cited the long standing notion that research is intended to guide current and future directions into best practices. They found that research that took into account cultural and ethnic factors as significant influences on student achievement were primarily a phenomenon of the last decade. Once research began to include culture as factor, this variable initially began to be considered as static within CLD groups. Arzubiaga et al. (2008) asserted that culture was a fluid variable with nuances within ethnic groups depending on acculturation and assimilation. They suggested that research on CLD groups should incorporate interactions of the subjects as well as their interactions with variables within the setting to be more reflective of current trends and practices and more accurately guide future directions.

In 2008, Harry reviewed several research studies, journals and books to determine the nature of the collaboration between CLD parents of children with disabilities and the 
professionals working with them. The author acknowledged that one of the mandates of IDEA was parent participation. With that in mind, Harry (2008) listed six examples of ideal communication between the home and the school envisioned by research. These examples included: (a) parents actively engaged in educational activities, (b) ongoing communication between home and school, (c) parents giving their time at school (d) parents providing support at home as in homework completion and/or supervision, (e) parents participating in school organizations such as Parent Teacher Association (PTA), and (f) parents serving as a bridge between the school and the community. IDEA embraced these examples and made them the core of its foundation and has reaffirmed this belief by strengthening them with each re-authorization. Harry (2008) further noted that CLD families' perceptions of collaboration may be more difficult to discern because they were being studied with traditional paper pencil methods. The author concluded that though much has been done to identify the collaborative nature of CLD parents of children with disabilities, it is still a work in progress. Therefore, schools should continue striving to understand parents' ethnic backgrounds, language and culture to facilitate the collaborative process for the benefit of children's educational success.

In 2008, Skiba et al. conducted a literature review of previous research in the field of special education with minority groups. They were searching for evidence of equity with different groups of learners. The authors examined factors such as disproportionate representation, test bias, economic disadvantage, educational opportunities, eligibility and placement as well as behavioral expectations. Skiba et al. (2008) cited different studies in search of data on the disproportionate representation of minorities in special education. Regardless of the formula used, the overall percentage of minorities identified as having a 
disability was grossly unbalanced compared to their numbers in the general population. With regard to test bias, the authors also reviewed numerous studies and concluded that standardized tests themselves had cultural bias embedded within the test items that would affect the results for children from minority backgrounds. Economic disadvantage was found to be a factor only to the extent that limited resources led to limited opportunities and limited experiences to increase knowledge and prior knowledge, all of which were measured by standardized tests. Eligibility and placement was closely linked to behavior in that the authors provided evidence to support the notion that minority children were more frequently referred to the special education tract due to behavioral difficulties. Also, they were more likely to be described as having difficulties learning, hence their referral for testing. Once there, the team was more likely to proceed with testing and eligibility for these children. Therefore, Skiba et al. (2008) concluded that based on the research, there was sufficient evidence of limited equity in special education with minorities.

Also in 2008, Trent, Kea, and Oh reviewed the literature in search of evidence that pre-service personnel were adequately being prepared to work with CLD parents. They discussed major national trends such as NCLB and IDEA that were introduced to reform education and involve parents in the educational process. These reforms were introduced during a period of tremendous change in the U.S. where CLD populations were increasing according to Trent et al. (2008). The authors remarked that the Association of Teacher Educators (ATE) and the National Council for the Accreditation of Teacher Education (NCATE) have made recommendations for increased diversity in the content of teacher preparation programs. The literature reviewed revealed that more studies were being conducted with CLD groups to add to the body of knowledge 
previously available. It further revealed that cultural concepts should be embedded within entire preparation programs to ensure that school personnel were sufficiently knowledgeable and prepared to engage parents of CLD children with and without disabilities.

\section{Locus of Control}

Although not originally part of this study, locus of control was one of the core concepts that emerged from the data analysis. A post-interview review of the literature revealed that locus of control can be defined as the ability to identify circumstances as being under one's own influence (internal control) or as being under the influence of others (external control) (Hamlyn-Wright \& Draghi-Lorenz, 2007). These researchers studied the impact of locus of control on three different groups of parents. They estimated that parents of children with the most severe disabilities would exhibit the highest levels of anxiety and depression, followed by those with developmental disorders in the middle. Finally, the parents of children without any developmental disabilities would exhibit the lowest levels of depression and anxiety. In order to verify their estimations, the researchers mailed out questionnaires to three groups of parents: those with no developmental disabilities, those with Down's Syndrome, and those with Autism respectively. Questionnaires returned were analyzed using statistical analysis methods. Results supported the authors' estimations. Further analysis revealed that locus of control influenced not only parental depression and anxiety, but it also had an impact on the stress of parents with disabled children. However, it only influenced the anxiety and depression of parents with non-disabled children. The authors remarked that parents of 
children with disabilities would likely need assistance from professionals to manage their anxiety and depression levels.

In an attempt to explain children's poor social behaviors, Swick and Hassell (1988), studied parents' competence and level of adjustment as a contributor to children's social competence or lack thereof. The researchers cited previous research linking parents' locus of control to children's behaviors. This study sought to analyze parents' efficacy and its resulting impact on the social competence of their preschool children. The questions that the researchers attempted to answer were: (a) Are parents' assessment of their children's social competence related to their own locus of control? (b) Are teachers' assessment of children's social competence related to their parents' locus of control? (c) Are parents' assessment of their children's social competence tied to the parents' perceived supports? (d) Are teachers' assessment of children's social competence tied to the parents' perceived supports? (e) Are parents and teachers' assessment of children's social competence similar? and (f) Is there a connection between and among parents' locus of control, parents' perceived supports and children's social competence?

The sample included children from diverse socioeconomic and racial/ethnic groups in South Carolina. Swick and Hassell, (1988) collected data in both individual and group situations using a locus of control inventory. Results found that: (a) parents' assessment of children's social competence was related to their own locus of control; (b) teachers' assessment of children's social competence was not linked to parents' locus of control; (c) there was a weak link between parents' perceived supports and their 
assessment of their children's social skills; (d) teachers' assessment of children's social competence was linked to parents' perceived supports; (e) parents and teachers' assessment of children's social competence were similar; and (f) there was a strong link among and between parents' locus of control, parents' perceived supports and children's social competence. Swick and Hassell (1988) stated that parents' locus of control and their perceived supports exerted strong influence on children's social competence in the early years.

Fulgosi-Masnjak, Gustovic-Ercegovac, and Igric (1996) conducted research on parental perceptions of their competence to deal with stressful situations. The authors used a Likert scale to measure parents' perceptions of their competence, their selfrespect, and their locus of control. The scale was used with two different sets of parents. One group had children with no developmental disabilities. The other group had children with mild developmental disabilities. Both groups of children attended the same school within an integrated setting. Results indicated that parents of non-disabled children expressed feeling more competence in their parental roles. In the category of self-respect, parents of non-disabled children, similarly felt more confident as individuals and expressed more pride in their accomplishments. Lastly, in the category of locus of control, parents of children with developmental disabilities attributed problems to external forces such as luck and destiny. The authors indicated that professionals needed to be aware of parents' perceptions of their parental roles, their self-respect, self-esteem and locus of control. These factors influenced parents' coping mechanisms and their ability to sustain engagement in their children's lives. 
The impact of parental involvement was explored further by Flouri (2006). Flouri reported on a longitudinal study that followed a group of individuals born within one week of each other for more than a quarter of a century. The intent was to identify what role parental involvement, locus of control and self-esteem played in academic progress. Participants were all born in Britain and were interviewed at various intervals until the age of 26. Data was collected using questionnaires and other documents. Over $90 \%$ of the participants were Anglo. Findings revealed that children, whose parents were involved by age 10, experienced increased educational achievement. This increased educational achievement was highly correlated to both self-esteem and locus of control. Secondly, locus of control and self-esteem were inter-related. However, fathers' involvement demonstrated a minor influence on male educational achievement but a stronger influence on female educational achievement. Finally, internal locus of control and parental involvement, especially that of mothers positively influenced academic achievement by the end of the study. This research helped to solidify the link between parents' locus of control, their involvement, and student achievement.

The findings of the studies conducted by Swick and Hassell, (1988), FulgosiMasnjak et al. (1996), Flouri, (2006) and Hamlyn-Wright and Draghi-Lorenz (2007), revealed that locus of control played a significant role in how parents perceived themselves, how they perceived their children, how they interacted with the school and how they reacted to the challenges encountered as parents. The parents' locus of control was a lens through which they not only assessed their skills and abilities, but also those of their children. Fulgosi-Masnjak et al. (1996), Hamlyn-Wright and Draghi-Lorenz (2007) 
also found that parents of children with disabilities exhibited higher levels of stress, anxiety and depression, felt less capable in dealing with the challenges of raising children with disabilities and had a greater propensity to operate from an external locus of control. The results provided a rationale for addressing parents' state of mind in addition to educating children, especially, if the children were disabled.

These studies presented parental involvement as one component within a myriad of variables parents encountered in the education process. They also explored locus of control and how it influenced not only parents' perceptions, but also their behavior toward their children and ultimately their involvement in their children's activities, including school. Authors such as Harry (1992a, 1992b, 2001, 2008), Palmer et al. (2001), Wathum-Ocama and Rose (2002) and Arzubiaga et al. (2008), Cogan and Oka (1992), Fulgosi-Masnjak et al. (1996), Flouri (2006),, Hurford and Cole (2002), HamlynWright, Draghi-Lorenz and Ellis (2007), explored parental interaction with school personnel, parental perceptions, parents' knowledge, attitude and engagement within CLD parents of children with disabilities as well as how locus of control had influenced parents' interactions with their children and the schools. Overall, the authors found differing levels of engagement and concluded that professionals within the education institutions were lacking in their methods of engagement with parents and failed to adequately assess or address the specific needs and concerns of CLD parents of children with disabilities. Hence, these authors concluded that though research had provided insight, the knowledge had so far not been translated into practice sufficiently to effect change. Finally, the authors recommended personnel preparation programs include a 
specific strand on parental engagement with the end result of increasing parental involvement.

\section{Chapter Summary}

Overall, this chapter has summarized the research regarding parental involvement within the general education population, minority groups, CLD groups and finally, CLD with disabilities groups. Based on the literature review provided thus far, parental involvement has been clearly identified as a key ingredient in student achievement regardless of the other factors present. This chapter outlined the challenges of parental involvement within each of these groups and provided examples of documented strategies that have increased parental involvement with each of these groups of parents. Research has been conducted with various minority and CLD groups with disabilities. Within each group, there were challenges presented with regards to parental involvement. A missing component of the research is whether all CLD groups faced similar challenges. This question has not been answered so far. The research on CLD groups has been conducted by researchers who identified with the group studied both culturally and linguistically (Harry et. al., 1999). The literature reviews revealed that parental involvement was closely aligned with parental culture, values and beliefs. It also revealed that parents' perceptions of their children influenced their interaction with those children as well as their interaction with the school.

The proposed study extended the body of knowledge by conducting research with an additional CLD group of parents who have children with disabilities. It explored parental perceptions of Haitian parents of children with disabilities. The focus of the research was to identify how Haitian parents of children with disabilities perceived the 
education their children were receiving, what role, if any, they saw themselves playing in this process and how they perceived their own children as a result of the identified disability. Finally, this research study sought to identify the supports and/or barriers Haitian parents said were impacting their involvement. 


\section{CHAPTER III}

\section{METHODS}

This chapter reiterates the research question and sub-questions. It presents the characteristics of the sample along with a detailed description of the setting, sample, procedure, and research design used as well as why this design was appropriate for conducting this type of study. It provides a description of how the data obtained was analyzed and categorized into the format that led to the theories formulated. The chapter concludes with a description of the role of the researcher and provides background information on the researcher relevant to the topic of the research.

Several authors have conducted studies on parents of children with disabilities to better gain an understanding of the level of their involvement with their children's education, their perceptions of the children and their views on the school system (Harry, 1992a; 1992b; 2001; 2008; Harry et al. 1995; Harry et al., 1999). These studies overall concluded that: (a) parents mistrusted school personnel, (b) parents felt that the school setting was cold and uncaring towards them, (c) parents deferred decisions to school personnel even when they did not agree with those decisions, (d) parents felt overwhelmed by all the written communication they received regularly from school personnel regarding their children, (e) culture had a strong influence on parental perceptions of children, (f) engagement with school personnel was limited to a large degree by language barriers and to a small degree by cultural differences, (g) parental involvement decreased over time because parents had limited understanding of the structures, guidelines and procedures of IDEA, (h) professionals had limited 
understanding of the minor nuances within a group's cultural and ethnic beliefs, and therefore, failed to engage them appropriately, and (i) parental involvement was strongly influenced by values, beliefs, customs and conceptual knowledge that were closely aligned with culture and acculturation.

This study's purpose was to extend the body of knowledge on parental perceptions of children with disabilities by examining Haitian parents. Trent et al. (2008) recommended that more cultural concepts be embedded within personnel preparation programs in order to increase the number of professionals prepared to engage parents of CLD children with disabilities. In addition, Harry (2001) stated that parents' perceptions of disability were shaped by distinct social norms. Harry (2001) further stated that although similar racial/ethnic groups shared common perceptions of disability, the source of the disability and ultimate value placed on the individual with a disability was directly related to cultural norms and expectations. Furthermore, Harry $(1996,2001,2002)$ discussed the role of the researcher in conducting research within cultural groups because these groups would not be readily studied by the mainstream culture due to language and cultural differences. The present researcher's role and background are discussed later in this chapter. Her qualifications put her in a unique position to conduct this study to provide additional information on Haitian parents of children with disabilities as the third largest minority group within the district where the study was conducted.

The research question that was investigated in this study was Haitian parents' perceptions of their children with disabilities. Specifically, the researcher sought to answer four sub-questions with regard to Haitian parents of children with disabilities. They were: 
1. What are Haitian parents' perceptions of the education their children are receiving?

2. How do Haitian parents perceive their role in the education of their children with disabilities?

3. How do Haitian parents' perceptions of their child's disability influence their relationship with their child and their involvement with the school?

4. What do Haitian parents see as supports and barriers to their involvement in their child's education?

\section{Setting}

This study was conducted in the largest school district in Florida, which is the fourth largest school district in the nation. This district was also among the most diverse nationally with regard to CLD populations. Data available from the district statistical highlights in 2008-09 revealed that the district served some 345,150 students in that scholastic year. A breakdown of the total number by race/ethnicity showed that Hispanic was the largest group of the population with $62.5 \%$ of the students. Black Non-Hispanic students accounted for $25.7 \%$ of the population, the second largest group. Anglo or White Non-Hispanics represented $9.1 \%$ and Other was $2.7 \%$. The percentage of students enrolled in special education was approximately $16 \%$ of the total number of students enrolled. Haitians were not identified as a separate group, but included in the data for Black Non-Hispanics. In order to get a sense of the number of children of Haitian decent in the district it was necessary to extrapolate the information from district's summary data available for languages other than English used at home. Of the more than 10 languages represented in the district, the percentage of students using Haitian-Creole as a 
language at home was approximately $5 \%$ of the population and was the second largest group after Spanish. French was the third largest with $1 \%$ of the school population. It is noteworthy to mention this because some Haitians identify French as their home language since it was the official language of Haiti along with Haitian-Creole.

At the time of the study, the district was divided into six regions. The study was conducted with students who attended public schools within one of the regions in the northern part of the district. The study was restricted within one area because of several factors. First, after careful study of data available in the district trend analysis of its population, the researcher selected this region to conduct the study because it had a large population of Haitian-Creole parents with children in both the general education and special education program. A review of special education programs and their locations in the district also revealed that several schools in this region had programs for children with disabilities ranging from mild to severe disabilities. Therefore, the number of parents needed to complete the research was attainable within the population available. Additionally, the district granted permission for the study to be conducted within schools in close proximity to limit researcher exposure to confidential student records in light of the fact that the researcher was also an employee of the district. Next, the close proximity of participants in the sample limited the impact of other variables such as economics and area of residence from having an adverse impact on the results. Finally, the study called for participants to be of a particular ethnic group, and this region had a large number of Haitian parents, thereby increasing the pool of potential participants who might have agreed to take part in the study. 
Special education is a process guided by IDEA mandates that flow from the federal to state and eventually to local districts. The guidelines become more prescriptive from the federal to the district at each descending level. Schools implement programs based on district guidelines. The federal mandate requires parental involvement as part of the decision making process. This district provides services to children with disabilities on a continuum from the least restrictive setting to the most restrictive based on current levels of functioning, student progress, parent input and the recommendation of team members (i.e., teachers, counselors, psychologists as appropriate, therapists as appropriate and school administrators). The most restrictive settings translate into programs where students with disabilities are secluded throughout the day in a special class with teachers providing specialized instruction to address their needs. This setting is referred to as self-contained by the district. The median settings provides part time assistance from specialized teachers for selected subjects to address delays in specified subjects (usually reading and/or mathematics). This setting is referred to as resource by the district. Finally, the district provides the least restrictive setting which offers support to students with special needs within the general education classroom in two ways. The first way is with a specialized teacher coming to provide assistance within that setting (collaboration). The second way is consultation between the general education teacher and special education teacher on minor modifications to the curriculum for students with disabilities (consultation). As mandated by law, each school must schedule Individualized Education Plan (IEP) meetings at least annually or more frequently, if necessary, to review present levels of educational performance and needs as well as goals 
for the following year. The law also mandates that parents be provided with written notification of these meetings in their language of proficiency.

A review of the district procedures manual outlining the guidelines for placement in special education reveals that this district uses categories to identify children with disabilities. The criteria are determined by Intelligent Quotient (I.Q.), present levels of academic performance at time of evaluation or re-evaluation, independent functioning, if warranted, social and emotional skills as appropriate and teachers' observations. This study focused on parents of children within the mild to moderate range of disabilities. The children's disabilities ranged from mild academic delays due to learning disabilities to moderate cognitive deficits with or without behavioral difficulties.

\section{Sample}

The criteria for participation in the study were the following: (a) the parent had a child with a disability, (b) the child was attending a special education program (ESE) within the public schools, and (c) the parent or guardian was the primary caregiver. The researcher deduced that the presence of Haitian parents of children with disabilities would be commensurate with their size in the region where the study was conducted. The size of the sample consisted of 10 Haitian parents of children with disabilities of various ages. The children's disabilities ranged from mild to moderate. The parents who agreed to participate in this study had children who were within the following categories: (a) six were in the mild range, and (b) four were in the moderate range of disabilities (see Table 1). All the participants interviewed for the study were the biological parents of the children. The children were enrolled in five different schools. Four children were in senior high school and six were in elementary school at the time of the interviews. 
Table 1

Data on Participant Children

\begin{tabular}{lccccl}
\hline Child & Gender & Exceptionality & $\begin{array}{c}\text { Current } \\
\text { Grade }\end{array}$ & $\begin{array}{c}\text { Current } \\
\text { Age }\end{array}$ & $\begin{array}{c}\text { Level at } \\
\text { Placement }\end{array}$ \\
\hline \#1 Adam & M & SLD & 1 st & 8 & Elementary \\
\#2 Bob & M & EBD & 4 th & 10 & Elementary \\
\#3 Charlie & M & SLD & 1 st & 7 & Elementary \\
\#4 Denise & F & InD & 12 th & 18 & Elementary \\
\#5 Erica & F & SLD & 1 st & 6 & Pre-k \\
\#6 Frank & M & InD & 9 th & 15 & Pre-k \\
\#7 Gabrielle & F & InD & 9 th & 15 & Pre-k \\
\#8 Henry & M & TBI & 11 th & 17 & Middle \\
\#9 Issac & M & SLD & 3 rd & 10 & Elementary \\
\#10 John & M & SLD & 2nd & 6 & Pre-k \\
\hline
\end{tabular}

$S L D=$ Specific Learning Disabilities; $E B D=$ Emotional Behavioral Disabilities; InD=Intellectual Disabilities; TBI= Traumatic Brain Injured

Note. Names of children have been changed to maintain confidentiality.

The parents had diverse background experiences. They ranged in age from mid 20 s to late 40 s. They had been living in the U.S. from 10 years to 28 years. The parents had varying degrees of English language proficiency. Six parents requested the interviews be conducted in Haitian-Creole because it was their dominant language. Four parents were interviewed in English since they were fluent in both English and HaitianCreole. The researcher conducted the interviews in English after conversing with parents to ensure that parents understood the questions and concepts throughout the course of the interviews. One of these four parents was fluent in English only, having entered the U.S. 
as an infant. Their educational backgrounds ranged from middle school to post graduate school. All parents except one indicated the children with disabilities had been born in the U.S., and all the parents had at least one other child who was either older or younger than the child with disabilities. In four of the families interviewed, the child with disabilities was the first born. In the remaining six families, the child with disabilities was either the second or third child born in the family.

The children with disabilities had up to four siblings. In two instances, parents had one child who was married and living outside the home. The gender for the parents interviewed was two to eight, men to women respectively. The gender of the students with disabilities comprised of seven boys and three girls. The marital status for some of the parents was somewhat ambiguous from the responses given. One was married, but the spouse was living outside of the country. Three did not have a spouse in the home and had never been married. Two had been separated but did not reveal if they had ever legally married. One was separated, but had been legally married to the spouse at some point. Two had been divorced and the other spouse had moved, leaving them in the home with the children. One parent was married and still living with the spouse. This parent was the primary caretaker of the child with disabilities and worked flexible hours to accommodate therapies, meetings, and other needs for the child with disabilities along with a normally developing younger sibling. All parents were employed in various industries from blue to white collar. They were of different religious backgrounds as well. Six reported attending church regularly and one attended intermittently. Three parents did not attend church on a regular basis (see Table 2 for data on the parents). 
All the parents who participated in the study had children with a history in special education for a number of years ranging from 2 to 10 years. Eight of the parents identified how many years their children had been in special education during the course of the interview. Two could not recall the number of years spent in special education. The information for those two children was retrieved from school records. Based on data available, five of the students had been in elementary school at the time of initial placement in special education, four had been in pre-school, and one was attending middle school. Parents were asked to provide descriptive qualities of their children, including how they were being serviced by the district. Three of the parents interviewed identified the specific category used by the district to serve their children. Seven did not know or could not remember. This information was obtained from school records. At the time of the interviews, six students were in elementary school and four were in senior high school.

\section{Procedure}

For the data collection, 12 Haitian parents of children with disabilities were asked to participate in the study. Creswell (2003) suggested 6 to 10 participants are appropriate for qualitative studies using phenomenology as a basis for understanding a group of individuals such as this one. The method used to identify participants was purposeful sampling. All of the participants were Haitian-Creole parents who were born in Haiti. They had lived in the U.S. for various lengths of time. All participants had at least one child identified as having a disability and participating in a public school program. 
Table 2

Data on Participant Parents and Their Attributes

\begin{tabular}{lcccccccc}
\hline & & & Number of & Years & Marital & Language & Church & Educational \\
Parent & Gender & Children & in U.S. & Status & Proficiency & Attendance & Level & Occupation \\
\hline$\# 1$ & $\mathrm{~F}$ & 3 & 10 & $\mathrm{~S} 2$ & $\mathrm{HC}$ & $\mathrm{R}$ & $\mathrm{K}-8$ & Patient Care Assistant \\
$\# 2$ & $\mathrm{~F}$ & 2 & 15 & $\mathrm{~S} 1$ & $\mathrm{HC}$ & $\mathrm{R}$ & $\mathrm{HS}$ & Office Employee \\
$\# 3$ & $\mathrm{~F}$ & 4 & 18 & $\mathrm{~S} 1$ & $\mathrm{HC}$ & $\mathrm{N}$ & $\mathrm{K}-8$ & Child Care Assistant \\
$\# 4$ & $\mathrm{M}$ & 4 & 16 & $\mathrm{D} / \mathrm{S} 2$ & $\mathrm{HC}$ & $\mathrm{R}$ & $\mathrm{K}-8$ & Factory Worker \\
$\# 5$ & $\mathrm{~F}$ & 4 & 28 & $\mathrm{~S} 1$ & $\mathrm{E}$ & $\mathrm{N}$ & $\mathrm{H} / \mathrm{S}$ & Unemployed \\
$\# 6$ & $\mathrm{~F}$ & 5 & 18 & $\mathrm{D} / \mathrm{S} 2$ & $\mathrm{HC}$ & $\mathrm{I}$ & $\mathrm{K}-8$ & Housekeeping \\
$\# 7$ & $\mathrm{~F}$ & 3 & 27 & $\mathrm{D}$ & $\mathrm{HC} / \mathrm{E}$ & $\mathrm{R}$ & $\mathrm{Post}$ Graduate & Pastor \\
$\# 8$ & $\mathrm{M}$ & 3 & 25 & $\mathrm{D}$ & $\mathrm{HC} / \mathrm{E}$ & $\mathrm{R}$ & $\mathrm{College}$ & Communications Tech. \\
$\# 10$ & $\mathrm{~F}$ & 2 & 12 & $\mathrm{~S}$ & $\mathrm{HC}$ & $\mathrm{N}$ & HS & Patient Care Assistant \\
\hline
\end{tabular}

Note: Gender =Female, $M=$ Male, Marital Status (S1=Single, $\mathrm{S} 2=$ =Separated, D=Divorced, $\mathrm{M}=$ Married); Language Proficiency (HC=Haitian-Creole, $\mathrm{E}=$ English; Church Attendance (R=Regular, I=Intermittent, $\mathrm{N}=$ None), Educational Level $(\mathrm{K}-8=$ Middle School, HS = High School) 
The parents were interviewed using notes and tape recorders. Prior to beginning each interview, the researcher asked the parent the language of preference for conducting the study. The majority of parents preferred Haitian-Creole and was interviewed solely in this language. Four did not identify a language preference and were interviewed in English. One of these four parents used a combination of English and Haitian-Creole. Nine of the parents were interviewed at home. One was interviewed at a school during a teacher planning day. This venue was chosen by the parent and it was expressed to the researcher that the school was more preferable for the parent than the home to conduct the interview. The interviews were transcribed, with case notes taken during each interview. A journal of the researcher's observations and feelings during the interviews was also kept.

After securing permission from the district, the researcher contacted personnel in different schools who were overseeing ESE programs, provided the district permission to conduct the study and asked them to randomly send home consent forms to parents of children with disabilities. The researcher dropped off copies of the forms at the schools to be sent home. Arrangements were made with personnel at these schools to receive the forms once returned by the parents. Forms were distributed in elementary, middle and senior high schools with large populations of Haitian Creole students.

Of the forms left at the schools for parental distribution, one consent was returned completed with specified times and dates to be contacted to schedule the interview. The rest of the forms sent home did not come back to the school though they had been printed in both English and Haitian-Creole. The researcher proceeded to contact school personnel to follow up with the parents who failed to return the forms. School personnel provided 
the names and contact information for parents of children with disabilities. The researcher contacted parents from the lists provided at random. When the researcher reached parents by phone, she explained the rationale for the study, the possible use of the information and how she would maintain the confidentiality of the information obtained. She also explained how the confidentiality of the participants themselves would be maintained and the process for conducting the interviews. The telephone calls were made based on the emergency contact information available at the schools. The parents were called in the afternoons, evenings and weekend. Six of the numbers were disconnected or no longer in service. Three telephone numbers reached voice mails and messages were left, but the parents did not call back. When the parents were reached, the researcher explained her background, provided details on the research and asked if they had an interest in participating in the study. Two parents declined to participate. Two others asked that the consent be sent home with the understanding that it would be it would be returned to the school once completed. The researcher sent consent forms to participate in the study through the school personnel previously identified after determining what school the child was attending. Through this method, the researcher secured 12 agreements to participate in the study. After the consent forms were returned, the researcher then scheduled appointments to conduct the interviews with the parents. The appointments were scheduled based on parents' availability and flexibility.

School personnel provided demographic data such as the parents' names addresses, telephone numbers and home language from the information available within the district's computer database after being provided with the district permission to access this information and the parental consent to participate in the study. Other 
information provided by district database also included program eligibility, initial placement date, program or programs under which students were receiving services and the setting where students were being educated along with their ages and grade levels.

Of the 12 consents to participate in the study that were secured, 11 interviews were conducted. The findings of the study reflect 10 of the 11 interviews conducted. One of the 11 interviews was conducted within 2 days of the one of parent's numerous discharges from the hospital due to illness. During the interview, the parent provided minimal information on the child and could not recall the name of the school, the program, the teacher, the grade, chronology of placement, current services and needs, nor academic progress. Every question was deferred to the child who was present during the interview. The parent then referred the researcher to the spouse and provided a cell number for him to be contacted for information regarding the child. The spouse was not present during the interview and the researcher was informed he was at work. The researcher attempted to schedule appointments with the spouse by leaving messages at the cell number provided. Phone messages were not returned and the spouse interviewed initially could not provide any details as to the most appropriate day(s) and times to reach the working spouse. The information obtained from this interview was not substantial enough to be used in the study. Another parent agreed to be interviewed and an appointment was scheduled for it to be held. However, the researcher visited the home twice and the parent was not home. Each time the researcher was told by a relative in the home that the parent had unexpectedly been called to work. The researcher left a business card with contact information and the parent did not follow up. That interview was dropped. 
For the rest of the interviews, when the researcher reached the home, she introduced herself, reiterated the purpose of the visit, elaborated on what information the interview intended to investigate and her school affiliation. The researcher explained that the study would not have any immediate impact on the individual child's access to his/her education and that the research was intended to inform the field in general. The parents were reassured that all the information obtained would be kept strictly confidential and that findings would be reported as a group to both the school district and the university. The interviews were conducted using an interview protocol to guide the process. However, the researcher asked probing questions and allowed the interviews to evolve based on the responses given by the parents within the confines of the research questions. Parents were told that the interviews could be stopped at any time if they felt uncomfortable or became ill. They were further informed that the interviews would be tape recorded as an added measure of increasing the accuracy of the information obtained. Information such as name, length of time in the U.S., job title, marital status and number of children was obtained first. The interviews proceeded with questions on the parent interview protocol (see Appendix A). The researcher deviated whenever necessary to ask probing questions, to clarify responses or to delve deeper into a particular issue.

In order to lend organization to the interviews, questions were developed to guide the process. Primarily, the interview questions were developed based on research reported by Wathum-Ocama and Rose's study (2002) of Hmong immigrant parents' participation in their deaf children's education. These interview questions also resulted from a literature review; analysis of records; participation in meetings; and observations 
of Haitian parents during IEP meetings, parent workshops, and conferences. For this study, parents were asked to describe how they interact with their child with a disability, how they explain the child's disability to family, friends and others, and how they envisioned the child with disabilities in the future. Furthermore, they were asked questions related to their perceptions of the education their children were receiving, their involvement in their child's education and of supports and barriers to their involvement. The questions were designed to elicit in-depth responses from the participants.

\section{Research Design}

A qualitative research methodology using Advocacy/Participatory Phenomenology was used to explore answers to the questions asked. This type of research allows the researcher to conduct research that can lead to advocacy on behalf of the participants involved. It can be easily translated into an action plan that effects change in the lives of the participants and their community (Creswell, 2003). The research questions called for drawing conclusions on parental perceptions of their children with disabilities based on particular responses to key questions asked by the interviewer. All participants were asked similar questions intended to elicit multiple word responses. It was necessary to probe at times in order to get participants to express themselves with more clarity. Therefore, it was necessary to engage them in conversations even prior to beginning the questioning so as to develop rapport and create comfort with the interviewing process. This type of format was informal in nature and allowed participants to be more at ease and open in responding to questions. Because this type of information had never been obtained previously with this particular population, open-ended questions (Creswell, 2003) in a semi-structured interview format provided opportunities to 
elaborate or ask probing questions as necessary. This type of interview process allowed the researcher to delve into participants' emotions and feelings regarding the phenomena being studied (Strauss \& Corbin, 2007). This method is usually used to study issues that are minimally explored or to expand on the knowledge base. The interviews focused on how parents perceived their child with a disability, their perceptions of the education the child was receiving, ways, if any, how the disability had affected their relationship with their child, and their perceptions of the school system.

Once the interviews were completed, the researcher proceeded to transcribe the tapes. One-half of the tapes recorded in Haitian-Creole were randomly selected to be transcribed by another individual with knowledge and expertise in Haitian-Creole to increase reliability of the data obtained. All the Haitian-Creole interviews were converted to English. This process too was completed with two independent translators for one half of the interviews. This was done to increase the probability that the essence of the data did not change with the translation. The researcher had two different people enlisted to do these independent tasks in an effort to reduce bias. Aside from the language, the only requirement for the individuals used was that they had to have an educational background so that they would be familiar with the terms used. The individual used to check the accuracy of the transcribing had knowledge of exceptional students. The individual used to check the accuracy of the translations had knowledge of general education only.

\section{Data Analysis}

The interview transcripts were analyzed using a coding process in order to sort, compare and prioritize the data (Strauss \& Corbin, 2007). The coding process was done by hand. Open coding identified discrete aspects of the events and interactions observed 
and assigned codes to participants' responses. At this level, the researcher reviewed the transcripts and notes in search of key words used to describe a child with a disability and whether those words were referred to in different instances. In axial coding, which was the next level of coding, the researcher grouped the codes for instances which might have occurred in the same data sentence(s) into conceptual categories. The constant comparative method was instrumental in the analysis of the data. The open codes were refined and conceptualized into axial codes, and, eventually, into higher-level axial codes. Examples of open codes included parent participation in school meetings. The axial coding was used to define how parents saw their children functioning. The third level was selective coding which sought to identify core conceptual categories that could systematically be related to other categories. These different levels of coding eventually led to the formulation of theories about these Haitian parents' perceptions of their children with disabilities, their perceptions of their role as well as their involvement in the educational process and the barriers and/or supports that influenced their involvement.

After the interviews were completed, the researcher transcribed all the information obtained. All of the interviews that were conducted, including those conducted in Haitian-Creole, were typed into a database in English. The information was typed initially for each parent. The interviews were labeled with the numbers 1-10. The next step was to read over the information looking for common words, responses, and expressions. Once commonalities began to emerge, the researcher began to group them into patterns of responses, independent of the questions that were asked to elicit said responses. The groupings of similar responses led to categorization of the information. 
This was the open coding process. The next level of data analysis, which was axial coding, involved analyzing the categories and grouping them into concepts. The concepts were themes that grouped similar categories under one umbrella. These concepts helped to make sense of the data obtained. The axial coding was transformed into a higher level axial coding. Finally, core concepts emerged from the analysis of the open codes, axial codes, higher level axial codes and categories. This final step led to the broad conclusions made from the analysis of the data.

\section{Data Sources}

To increase internal validity of the data collected, triangulation was used. The information obtained from parents was compared to data from school records, parent and teacher meetings, and IEP meetings. Information from these sources provided insight into the parent-child relationship and the nature of parental involvement. The researcher secured parent permission to review district records and documents and attend any parent teacher meetings or IEP conferences that were held the course of the study, if feasible. These sources were used to corroborate information obtained from the parent interviews.

As an additional measure of corroborating information obtained during the interviews, Cumulative records (CUM) were reviewed by the researcher. The CUMs are maintained by all the public school system for every student. They follow students from kindergarten to 12 th grade and contain confidential information on students as well as parental information relevant to students' educational needs. This review was done to verify the accuracy of information provided by parents on student placement, psychological evaluations, and medical records if applicable, report cards, test results, parent contact information, student progress as well as interventions conducted with 
students, whether academic or behavioral. Review of these documents allowed for comparison of the information obtained from parents to information obtained by other sources regarding students' level of functioning, needs and abilities. It provided a platform for establishing how parents perceived their children. Parental permission to review these records was included in the consent to participate in the study. These records gave insight into parent participation in the meetings to discuss student progress and needs. These meetings must be held annually as mandated by law and can be held more frequently, if necessary. Parent notifications must be included in the CUM record. Therefore, parent signature or documentation of their response would indicate the level of their involvement in their children's education.

\section{Role of the Researcher}

The researcher, is who is a Haitian-Creole educator within a large urban school district for the past 19 years, conducted all of the interviews. Her qualifications stem from both formal and informal expertise. She has lived in the U.S. for the past 33 years. She arrived as a teenager and speaks Haitian-Creole fluently. Her formal expertise is that she has a Master's degree in special education. Prior to holding her current position as an assistant principal, she held numerous jobs in the field of special education within this district. These included: instruction of elementary school children with disabilities, parent training for the parents of these children at home and at school, translation during meetings, workshops, and psychological evaluations. She has also presented at conferences at the local and national level on the topic of children with disabilities. In her current assignment, she works in a school where the majority of the parents are of 
Haitian-Creole background. In an effort to reduce bias, she did not interview any participants from her school.

The informal expertise is that she dealt with Haitian parents of children with disabilities extensively within the district where the study was conducted. For more than a decade, she was involved with evaluation, placement and monitoring of programs for children with disabilities. During that time, she interacted with Haitian parents of children with disabilities during meetings at school, as a parent trainer, conference presenter and translator. The informal knowledge also stems from having a sibling with disabilities. This sibling is an older brother who lived with the family for the majority of his life, until he became a quadriplegic as a result of a stroke. The disability was never diagnosed formally as a child because there was no formal system in place to address the needs of children with disabilities in Haiti at the time. This disabled brother grew up with the family knowing that he was somehow different and yet part of the family. He stopped going to school somewhere around the first grade. Although, the researcher's parents emphasized education with the other siblings, the disabled one was not obligated to attend when he began to lose interest in school. The schools could not accommodate his needs nor individualize his learning. The expectations were different for him because he was perceived as being different. By the time he entered the U.S. with the rest of the family, he was already an adult. Prior to the stroke, he was functional and independent. However, he never managed to learn English and still communicates only in HaitianCreole. The formal and informal experiences gave this researcher the background and knowledge necessary to conduct this study on parental involvement with this racial/ethnic group. 


\section{Chapter Summary}

This chapter outlined the framework for the research study and described the procedures used to obtain the information as well as how the data were disaggregated and analyzed. It also included the rationale for the design. The research questions were revisited along with a detailed description of the setting, sample, procedure, research design, data analysis, and data sources. The chapter outlined who the participants were and how participants were identified and secured. The method in which the interviews were conducted was presented. The rationale for the research design was provided along with a description of how the data were organized, sorted, and corroborated. This chapter concluded with a description of the characteristics and background of the researcher and her role in the study. 


\section{CHAPTER IV}

\section{FINDINGS}

This chapter presents the findings of the study using information obtained from

the interviews, data from records, and field notes recorded during the course of the study. The information from interviews was sorted using organizational codes based on data analysis methods suggested by Bogdan and Biklen (2007) and Stauss and Corbin (2007). The parent responses were organized using open coding of the information into more than 20 codes such as initial eligibility and understanding of the process, financial issues, relationship with God, and medical issues, just to name a few. The open coding of the data subsequently revealed four axial coding categories which were: (a) home school collaboration, (b) parental concerns, (c) relationships and perceptions, and (d) whose fault. Finally, the axial coding translated into two conceptual themes that explained the findings of the research and answered the research questions. These themes were coping mechanisms and locus of control. Within each of the categories that emerged from the open coding, the issues that became relevant will be discussed further to provide insight into how the responses provided by parents resulted in the conclusions formed by the researcher. The themes, patterns, and ideas provided insight into the perceptions, concerns, needs, and priorities of Haitian parents of children with disabilities. They answered the research question and sub-questions which were:

What are Haitian parents' perceptions of their children with disabilities?

1. What are Haitian parents' perceptions of the education their children are receiving? 
2. How do Haitian parents perceive their role in the education of their children with disabilities?

3. How do Haitian parents' perceptions of their children influence

their relationship with their child and their involvement with the school?

4. What do Haitian parents see as supports and barriers to their involvement in their child's education? (see Figure 1 for a representation of the coding process).

\section{Home-School Collaboration}

This theme was organized using topics that included parental awareness and insight into the eligibility process, how helpful parents found the special education program to be, and what improvements they perceived in their children as a result of placement into the program. It also explored the nature of the interaction and communication between parents and schools and what concerns parents had along with their perceptions of supports needed from the school in the education of their children with disabilities. Finally, parental involvement at school and at home was explored. Parent responses gave insight into their perceptions of the school and their role in the educational process. Taken together, these responses revealed parents' perceptions of the school, the program and their role in the process.

\section{Initial Eligibility/Understanding of the Process}

This category provided parents with the opportunity to demonstrate how well they understood the special education process. Parents' understanding of the process was demonstrated by how they described specific components of the eligibility and placement 
into special education as well as their participation at various intervals. All parents interviewed commented on their understanding of the special education process and how their children came be eligible for participation. For example, Parent 7 expressed that she understood the process completely because an older child who is now an adult had been placed in special education. However, she also stated, "They told me she cannot function normally and that they are going to put her in a special program. I was crying and asking how come my son is so smart and my daughter is like that. I did not understand that." Parent 8 , whose child had suffered a traumatic brain injury also stated he understood the process and was part of it following the accident. He stated that there was complete collaboration between the home and the school during the entire process and that meetings were frequently held among the team members and the parent to discuss eligibility, placement and progress monitoring.

Six parents only explained the type of specialized services the children were receiving. Parent 3 said, "That means the subjects that he is not good in, he has a program to help him, in those that he is not good in." Parent 3 continued, "Because he did not completely understand, like he did not excel in the subjects that they were helping him in and he did not really understand." Parent 2 remarked, "There were programs being prepared in the school so that they could help him for me." Parent 5 said, "The only thing that I thought was, she is not the same as the other kids. She is not learning as fast as the other kids. That's what they told me. I think that's what it was." When asked about the eligibility process, Parent 4 responded to the interviewer with the following question, "The question I would like to as is, when she is finished with public school, are 
Organization of Data

\section{Core Concepts}

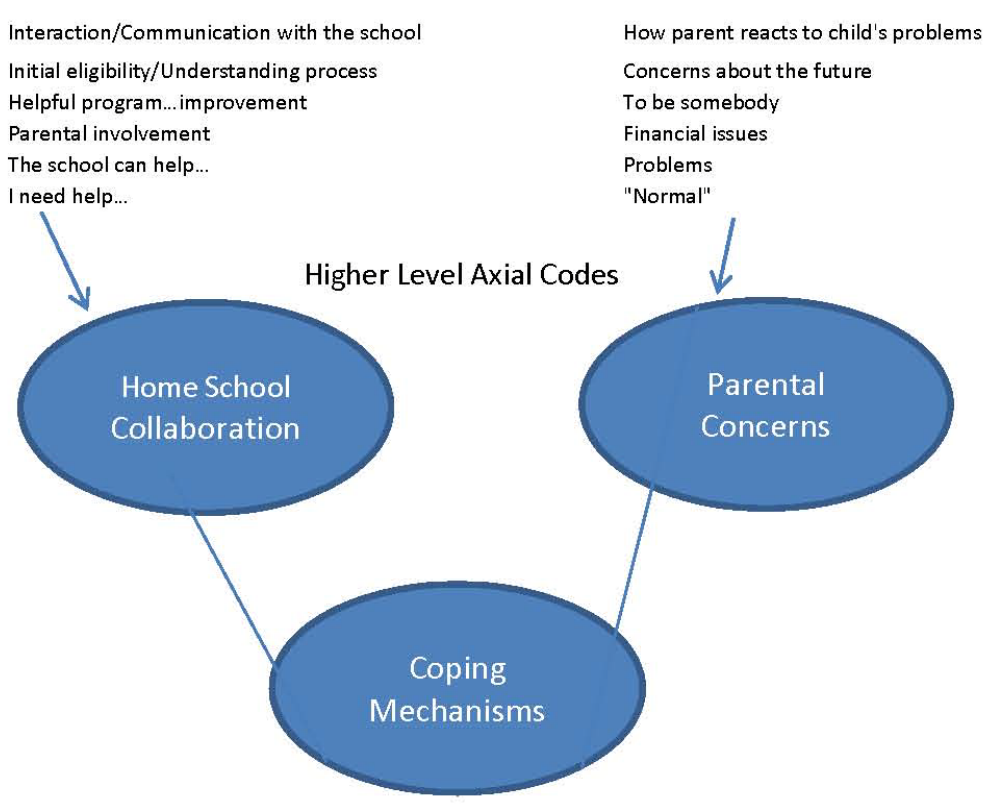

\section{Core Concepts}

Interactions with family/friends/community (same

Interactions wit

Something in the brain

Not the school's fault

Medical Issues

elationship with siblin

Home Behavior

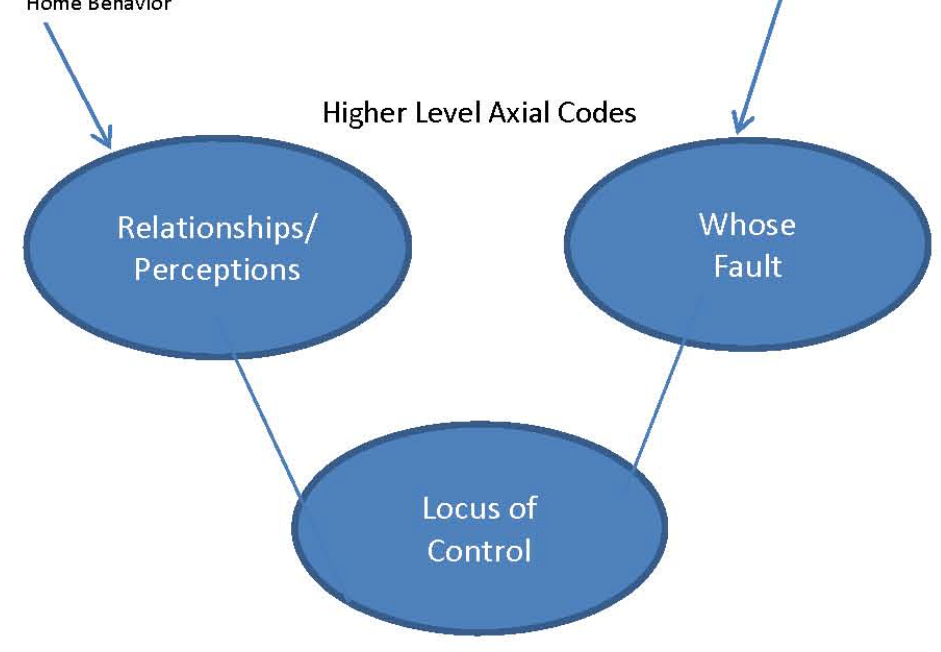

Figure 1. Organization of data. 
there any programs that she can continue in?" Parent 9 said "They helped me because they completed several tests on him." Parent 1 admitted she did not understand the process by acknowledging, "I did not really understand the process, no. They kept asking me a lot of questions. A social worker came to my house. They were doing a lot of tests for him." When asked about the parents' participation in eligibility and placement Parent 10 responded with:

Yes, I did, not but not the full extent. Now I know that he has some needs and that the needs have to be met and that to put him in a regular classroom was not going to help him or solve the problem. So I told them, "Ok, no problem, whatever you say."

The understanding that there was process that led to the children being identified as children with disabilities was not clearly evident in some of the parent responses. Some of the responses indicated that parents did not have a clear understanding of what special education entailed, although they were present at the meeting where eligibility and placement were discussed. Some of these parents knew that their children were being educated in different settings and that they had difficulties progressing through the curriculum. However, their comments seemed to suggest they did not have clear explanations about the process nor did they fully understand the rationale behind the initial eligibility and placement in special education. For example, Parent 9 stated, "They put him in, but did not tell me for how long." Although IDEA mandates parental involvement in the educational process for children with disabilities, these responses clearly indicated that the parents in these instances had very little understanding of their role in the process and lacked the knowledge to participate effectively. 


\section{Helpful Program/Improvement}

This was another category where parents expressed their perceptions of the program and their children with disabilities' participation in it. Nine of the 10 parents interviewed felt the program had been helpful to them and that their children had improved as a result. Overwhelmingly the parents felt that participation in special education had helped their children to progress academically. Parent 2 stated:

Yes, the program has changed what I think about him a lot because he gave me a proof of that. I was helping him to study the times tables.... he stopped and said, "Mommy, ask me a question and I will answer you." I asked him a question, honestly... He took a long time to count until he got the number needed for the answer. Anyway, he gave me the answer, despite everything, which means that he is trying.

This parent felt that the child's ability to reason was a direct result of participation in special education. This parent continued to say, "Yes, I see an improvement. On the contrary, even in his report cards when they come they surprise me," acknowledging that the grades were improving. Parent 3 stated, "Yes, the teacher is helping him, other people are helping him on the same day and it is better," when asked what impact she felt the program had on her child. This parent continued with, "They help him find more explanations and then what he does not understand, he is made to understand." Parent 3 made the following comment:

I am happy because he was not doing well. It is only now that I see him making progress little by little. Things like addition, subtraction; he did not know how to do them. Now he is a little bit better, which means the program is helping him.

Overall, parents stated they saw improvement in their children and felt the special education program was helping in enabling their children to access an appropriate public education. Parent 4 said, "Well the program is good for her because from where she was, 
if she was not placed in the program she might not be where she is now, you understand." Parent 10 made a statement that expressed only partial satisfaction. She said, "Well, it has helped him, but it's just the class. That's what I don't like, where he is being placed now. I don't think the environment really helps him.” Although she agreed her child needed a special program, she did not agree with the current setting. Parent 5 said she did not see any changes in her child as a result of placement. She said, "Nothing has changed. No, not really because she's the same person." When asked to elaborate on her comment, she continued with,

I said the same. I mean you know why I keep saying the same because, the same ways she does at school, the same way she does at home. Like, she doesn't listen, she doesn't sit down in her seat.

This parent expressed dissatisfaction with the school program, but her explanation addressed behavioral issues. However, this child was placed based on academic delays, not behavioral.

\section{Interaction/Communication With the School}

In this category, parents' responses gave insight into the nature and extent of the interaction and communication between home and school. Parents' comments provided information on the frequency of their interactions with the school, their perceptions of the school, and their ability to communicate with school personnel. All parents stated they communicated with the school. However, the frequency and intensity of this interaction varied widely from parent to parent. Parent 6 commented, "I go to the school when they call me, but you know my English is not so good." Parent 8 said:

Like I said, I keep track of everything he does. I will go to the school, talk to the teacher. Oh, every year, I will talk to all of them. I don't have any problem with 
doing this. Every time I am off, that's the first thing I do, jump in my car, go to the school. I think he likes this too.

Parent 1 stated, "They are always calling me to tell me he does not want to sit down. $\mathrm{He}$ is always touching other children." She continued to say, "Anytime, the school calls me, I am always available. I don't work during the day, I work at night. I work so that I am available when they need me.”

Parent 4 explained the nature of his involvement with this comment:

It is not easy for me to go to the school, but sometimes, I go, understand? If they invite me to a meeting for her, I always try to go. Sometimes, I call to ask information about her. I see that she interested in school. They never complain about her. She is not absent from school easily. That is one reason why I don't waste time to go check on her to see what she is doing at school. When they need me, they have meetings; they always invite me to go.

Parent 6, who discussed the challenges of communicating with the school due to language difficulties, stated:

I don't have anybody to talk to. When he was younger, the Haitian social worker was good. I used to ask her questions, talk to her. Since then, he has changed school and I have lost communication with her. I don't know where to find her. When you called me, I was happy because I found someone to talk to. There is a lady with a child similar to mine; her child is in many programs. She is not Haitian. She has a place for her child to go when he is 18 years old. I ask her how she found these programs. She tells me that she will explain to me how to get them. She has not had the time yet. I don't have anyone to ask questions, explanations about anything.

With Parent 7, her communication and interaction with the school was influenced by

other factors. She said:

I don't go to the meetings when they happen on Wednesday night because I am a pastor, but I call. I call not only for my daughter, but even with my son. He was 7 years old when we came here. I always talk to the teacher, sit in the classroom. I call and make sure they know me. I find out what is going on. 
However, Parent 9 said, "I go to the school for meetings, I have two jobs. Therefore, I am never at home." The responses were influenced by parents' perceptions of their role, children's difficulties, language barriers and other constraints on parents' time and resources. Parents' comments indicated they wanted to provide justification to explain the extent of their communication with the school. The responses indicated there was variability in the level of communication between parents and schools.

\section{The School Can Help}

This category sought to identify parents' perceptions of the support they required from schools in educating their children with disabilities. It also provided another opportunity for parents to comment on how they viewed the partnership between home and school. Of the nine parents who commented in this category, seven felt the school should assist them with their children both at home and at school by providing support services. Two felt the school should assist them, but did not specify what supports they needed. Parent 1 remarked, "I always tell them what problems I have and how they can help me with him because I don't like to hide anything he has." Parent 2 stated, "I was always thinking that if the school helped me honestly to find a place here. A place to hold him and monitor him (pause) that is what I was thinking if they had it."

This parent expressed this comment:

What I would like for the school to do is find, anyway, there is a process being conducted at school to transfer him to another school for me. I would like for the school to transfer him completely to a school that can help him. It is not that the school cannot help him; it is that for kids like him, this school cannot help him.

Parent 3 said, "I don't know what programs the school has that can help him. The more the school can help him, the happier I would be." The next set of parents was more 
specific on what the school could do to assist parents with the education of their children with disabilities. Parent 7 said:

I call the teacher; I explain to her what I need. The program they have at school is not enough for kids like her. They need more. I have to be on her back. I don't want to be on the teacher's back, so I try to do things with her on my own. I take her somewhere to experience real life situations. I tell her now, if you are here by yourself and you want to go home, what would you do? How would you get help? Would you get in a car with someone? Make sure you find a police officer and that is who you would ask for help. I show her what the police officer is dressed like.

Parent 8 made a similar comment regarding the adequacy of the services received from the school by stating this:

The school system should have people who do the follow-up of these kids, you know, up to the end, until the kids become better, you know, or if they cannot be better. Probably, there is another program, but when the kid is still in the school system, I think they could have done something, but when you have somebody who is taking care of the whole district this is not good. I am not going to say they should assign one person per school where you have those programs, but at least, a little bit more because of those kids.

I need help. In this category, parents were more specific in describing the type of assistance needed from the school in order to be more effective. Six parents said they needed help from the school. Four of the six were very specific in identifying their needs, while two were more vague. Parent 2 , who was waiting for her child to be moved to a more appropriate program stated, "Yes, I would like for the school to move faster. They take too much time. For me, I don't know if it's because I am the one who needs the help, but I find that they move too slow." Parent 4 said, "I would like for the teacher to do her part the way she is supposed to do it. I teach her family education. I know what my part is and I accomplish my part.” He further stated:

I would like for her to continue going to school. I don't know how if that can happen because I don't know a lot about the educational system here in this 
country or what can happen, if they can keep her. College and public schools are not the same. They are not governed by the same body. I might not be able to say anything, you understand? They don't give parents this information to know what is happening, what is going to happen. Anyway, my decision is for her to stay in school.

Parent 9 explained her needs with this statement:

The need I have is that I would like for them to check for better programs. I would like for him to stay at school, but I would like other programs too. I would like to know how I can do that. I would not like for him to stay home. I would like for him to be in programs all the time.

\section{Parental Involvement}

In this category, the researcher sought to identify instances where parents commented on the nature of their involvement with their children with disabilities, both in school and with homework activities. Nine parents spoke about the extent of their involvement with their children. The following was a comment made by Parent 5 when asked to discuss the nature of her involvement. "I don't know who to speak to, I don't know it it's the teacher, the principal, I don't know who to speak to."

Parent 3 said,

Well, most of the time, when he was not in an afterschool program, when he came home, I would try to help him. Sometimes, he did not want to do his homework. It is when I came home that I would help him. Now I put him in an afterschool program so that he can do his homework. When I come home from work, I go and meet the afterschool care bus that brings them back to the school.

Parent 7 described the nature of her involvement in this statement:

I call not only for my [disabled]daughter, but even with my son. He was 7 years old when we came here. I always talk to the teachers, sit in the classroom. I call and I make sure they know me. I find out what is going on.

Parent 8 explained this scenario: 
Basically, all the teachers know me because, like I said, I would go over there. I will talk to them, especially he had one.... So, I went over there.... I had to get everybody on board and meet with them. Probably, that is the only time I meet with them four times during the year. Oh, yeah, I ask for a meeting with them and then we sit down and talk about him. That was pretty hard for me to understand because, basically, I think that, that year if I didn't do it, he would have spent basically, the whole year with teachers who did not even know about his condition. For these kids, they need to know exactly what he is all about, what happened to him. Otherwise, they are not going to be able to understand him.

He described parental involvement in this way:

I am telling you if you don't know how to follow up or pick up the phone, you don't get the help you need, believe me. That's exactly my point. I believe when kids get this lost, if we don't do anything we going to have some serious problems in the future, and I believe like my son, lots of kids could have gotten better if they had people following them, you know, but that was not the case.

When it comes to assistance with homework, Parent 4 said, "I can tell that for helping her with homework, I do not know enough to help her. That is one of the problems that I have.” Parent 9 stated, "I have two jobs. Therefore, I am never home. I don't have time to work with them at home." Parent 10 discussed her frustration with what she perceived as work that was too difficult for her son to complete at home. She said:

I am like ok yes I understand that's him but there are ways of helping, but I feel like he is much better than this. They know I was more involved than this year. Even the teacher, she doesn't send homework anymore. When she sends homework, I say, you know, what's the need. She was sending homework that was not appropriate. So I told her no, you know I have my material. I am working with him. There is a process. I am not going to send them back, I am not going to waste my time, you know, to go over those things with him. They know.

\section{Parental Concerns}

The categories addressed under this theme were financial issues, parents' perceptions of "normal," parents' desire for children with disabilities to become somebody, how parents reacted to the child with disabilities' problems, concerns about the future, and parents' perceptions of the child with disabilities' problems. Taken 
together this theme fleshed out the overriding concerns parents of children with disabilities had and how these concerns influenced their daily lives. The fact that these concerns impacted daily life also meant they influenced parents' perceptions of those children.

Financial Issues

Five parents commented on their limited resources and how it had exacerbated their difficulties. These parents described the financial hardships encountered in taking care of a child with disabilities. Parent 6 stated, "What I know is that kids who are born like him, the state helps them (financially), but he does not get anything." Parent 9 remarked, "I am very busy because I must work. Their father does not live here to help me. I work different hours at two jobs as a nursing assistant. Sometimes, I leave one job and go to another." Parent 7 discussed the medical aspect of taking care of a child with disabilities with the following:

No, that is what I am telling you, they don't give her Medicaid because they say I make too much money. They don't give her anything. That is what I mean, they should have coverage for her. I have to come up with money for her. But now, her father covers her.

Parent 4 discussed how financial pressures affected his parental involvement with this comment:

Allow me to tell you that, I am a single parent. The salary that I earn is so minimal, since I am the only one working that's it. Since I have a family to take care of, I become a slave to my job. For me as a parent, I see that I cannot take a day off to go to the school. It is not easy for me to go to the school, but sometimes I go, you understand?

Parent 2 explained her decision to send her son to Haiti in this way, "I found myself in a situation where I was obliged to send him to Haiti form time to time for a few months. 
No one has money, my mother and father are old and I am working." This parent was the one supporting her elderly mother and father who reside with her along with her disabled son.

\section{"Normal"}

One method used to categorize parents' perceptions of their children was the word "normal." Parents used it to describe children's behavior, their perceptions of progress as well as the long term outcomes they had for those children. Five parents commented on their perceptions of what constituted as "normal," and how their children fit within its spectrum. Parents' perceptions of normal were extracted from their specific comments regarding their children's behaviors. Parent 1 said:

They told me he is normal. For me, he is not really normal because he never sits down quietly. When you say something to him, he shows that he understands. After a little while, he does not understand.

This parent perceived her child's limited attention span and inability to follow directions as indications that he was not normal. Parent 10 described her vision for her child in this way:

You know, I have been picturing him as normal, he probably will not be one of the best students or one of the brightest, but I feel like, you know, he will be functional. He is going to function in a regular environment. He will have his little things to overcome, but you know, he will do fine.

When we delved into parental perceptions of the children with disabilities as adults, Parent 2 said, "Only I would like for him to grow up normal." Parent 4 stated, "I am not saying she is a normal child. She is able to do what she needs to do. She cleans her room, she even cooks. She does everything." He discussed his perception of what normal meant in relation to his child with this statement: 
The problem is only her difficulty learning. She does everything else normal. That means that what the other kids are doing she is not able to do academically. She does everything else in the house like cleaning, taking care of herself.

\section{To be Somebody}

This was another term used by the patterns to describe their aspirations for their children. There were four parents who commented in this category. Parents expressed their expectations for the children with disabilities in similar terms. They expressed their aspirations for these children in common terms. Parent 1 said, "I would like him to be somebody in this country. He was born in this country. I would like him to be

somebody." She continued with, "I would like for him to be somebody when he grows up like everybody else. I would like for him to have all his senses like other people.” Parent 4 expressed his concern for the child with the following:

You know that sometimes when you are from a parent or family that is poor, there certain things that you think of but it is difficult to really do them. When you are poor and you get to a certain age you cannot always help your kids. That is why I would like for her to help herself to become someone, because I will not always be able to help her.

He elaborated with:

Well, what I was expecting from her before she was placed in the program, I was expecting her to perform well in school, become a high level person, become a Ms. so and so like you, but based on what I see, she will not be able to accomplish that. I am obliged to accept it.

\section{How Parent Reacts to Child's Problems}

Five of the parents explained how their children's disabilities had affected them personally. Parents described the impact of dealing with these children in different ways. Some of the parents mentioned experiencing stresses that were physical in nature. Parent 1 said, "Well, it is the way he is living that bothers me." Parent 6 discussed being so 
stressed that she developed a physical reaction. She described her reaction to her child's behavioral difficulties at home with, "Sometimes he makes my head hurt. Sometimes I have to go out." Parent 8 explained how he felt at meetings where the child was being discussed following the accident that resulted in the child becoming traumatic brain injured. He said:

I had problems and I was crying explaining to them, sometimes, I had to pause, you know, to give myself the time to compose myself and start again, but after a certain time, looking at it, seeing exactly what it is, I say hey, it become easier for me, but I think other things they can do too, get some program where parents can go over there and listen to others addressing those issues.

This parent also expressed his perceptions of how other parents in the Haitian community react to having a child with a disability. He said:

That is the kind of thing I see in the Haitian community. They are afraid to talk about it. You have got to see the kid is slow, you say it. That's what's going to help you. You go out there and you try to find help for them, you know. You just don't sit down and hide it. I am helping my kid. I am not hurting anybody.

\section{Concerns About the Future}

In this category, parents expressed their greatest concerns for the future in regards to their children with disabilities. Nine of the parents stated they were concerned about their children's ability to succeed in the future. Parent 1 said, "Tomorrow, if he is not able to function in society, he will be like people who are lost, worthless." Parent 2 was concerned about her child being retained. She said, "I hope that he does not repeat any classes, that he understands what he needs to do to move forward." However, overall, their comments were broad. Parent 4 said this: 
Well, what troubles me the most is when I think of her. Because you know in this day and age in this country that we are living, if you don't have an education, you might not be able to live a comfortable life like everyone else. This is the problem that I have with her. I would not like for her not to have a good job, for her to suffer because of the level of her education, That is what I would not like.

Parent 7 discussed her greatest fear with this comment:

Like I don't want to leave her home by herself. I don't want anybody to harm her. I don't want to send her anywhere. I will send her to my cousin, but I pray in the future she will be able to function on her own. One day, she can have a job, balance her checkbook. Just the little things. I don't plan on being with her the rest of her life. She needs to be on her own also. To be able to live on her own.

Additionally, Parent 5's stated goal for her daughter was: “To finish school, like finish high school. I am hoping that she passes her class and finishes high school and college and stuff like that."

\section{Problems}

Four of the parents discussed their children with disabilities in terms of the problems they encountered with them. Parent 1 said, "A child like that, that is giving problems, I would like for him to find a special school." She was referring to the behavioral difficulties that the child exhibited at school. Parent 4 described his daughter with, "The problem is only her difficulty learning. She does everything else in the house like cleaning, taking care of herself." Similarly, Parent 9 identified her son as having learning difficulties and said:

He is trying. He is trying harder because he is starting to learn how to read. He did not know how to read before. That was his main problem, but now he reads a little. Now, he can read a little, before, he could not read at all.

Parent 7 explained how he dealt with problems encountered with his son:

Yes, it is difficult at time, but what I am saying is, I don't try to put any pressure on him because I know it's not going to be good. As a matter fact, I have been told by the doctor, "You know, we don't need to put any pressure." 


\section{Relationships/Perceptions}

A third theme that emerged in the analysis of the data was that of parents' relationships and perceptions. Parents' relationships and perceptions provided the framework for how parents viewed their relationships with God, the child with disabilities' relationships with siblings, the nature of the interaction with family/friends/community as well as whether they considered the child with disabilities to be the same or different from peers and/or siblings. Finally, the parents discussed the home behavior exhibited by the child with disabilities and whether the difficulties stemmed from the child's attitude versus aptitude.

\section{Relationship with God}

Seven of the parents referred to their relationship or belief in God in discussing their children with disabilities. Parent 1 talked about her son's behavior and said, "Only God can say something about how he acts." She continued to describe how she had enrolled him in a behavior modification program by using part of her rent money for 1 month because friends and neighbors had told her the program would be effective in addressing his behavioral difficulties. Yet in the end she stated, "If there is something to be done about his behavior, God will bring about this change in him." For Parent 2, who was looking for an alternative program to meet her son's needs, said, "If the school wants to help me in other ways, I would give thanks to God.” Parent 7 framed her hopes and dreams for her daughter by saying, "To be able to read, to be able to read left and right, to touch and not to touch because it is really dangerous. This is what I would like to happen. I pray, I believe in God. I pray for it.” She continued to discuss her daughter's current 
level of functioning by stating, "Thank God she is like that, I see some other kids who are worse than her, but thank God, I know that I cannot cover every area, but I feel like whatever I can do". These parents' comments suggested their relationship with God was used as a coping mechanism to deal with the challenges encountered in raising children with disabilities.

\section{Relationship With Sibling(s)}

In this category, four parents discussed their observations of the interaction within the household. Parents described the role siblings played in supervision and care of the child with disabilities as well as how the siblings interacted with each other. Parent 1 said these words:

Yes, when I am there he [disabled child] is watching me to make sure I don't see when he is hitting her. He is mean to her. It affects me. His little sister is like a computer. When Adam is doing bad things, she thinks they are good things and she copies him.

Parent 6 relied on the siblings to assist with supervision of the child with disabilities. The parent said, "Only his brothers and sisters and I that I leave him with. Only if his brothers and sisters are going to be there as well will I let him go." Parent 8 whose child was diagnosed following an accident stated:

Everything has changed because, you have to understand. I am not going to say he is $100 \%$ the way he was, life will never be the same anyway, but the life of the family has changed because we have to adjust because we got two others, one brother and a sister. So we kind of had to tell them, explain to them how to handle him. They couldn't understand because they are little. That part was difficult. It's still difficult, you know, because you have to make them understand, hey listen, he is older that is true, but he has some problems. Everybody needs to pull together to help him. But don't forget they are kids. It is difficult for them at times. 


\section{Interaction With Family/Friends/Community (Same and Different)}

Nine of the parents discussed how the child with disabilities functioned within the family unit, among friends and within the community. Parent 2 discussed how she sent her son periodically to Haiti when overwhelmed by his behavioral difficulties. She said:

I found myself in a situation where I was obliged to send him to Haiti from time to time for a few months two times a year. When I send him to Haiti, I have my brother to watch over him for me other there. Honestly, every time he comes back there is a little change[improvement].

Parent 6 discussed how her son's behavioral difficulties left her reticent about sending him to spend time outside the home. She said, "He only stays with us. I don't let him go to other people's houses. I don't send him to other people's houses. They might not tolerate him." Parent 4 also provided insight into the topic of having a child with a disability by saying, "Only her siblings know that she is in a special program."

However, there were parents who stated they felt comfortable in allowing their children with disabilities to interact with the larger community and wanted these children to be included. Parent 7 discussed her daughter's participation in church activities with:

She is a normal 15 year old. She does everything. She handles everything. She knows all the keys. She watches what is going on. The other thing I think people can see about her, you know, during bible study, when they ask questions, she walks around, she does not sit down. They don't ask questions about her behavior and they don't force her to do anything because I think they know.

Whether parents regarded the child with disabilities as being the same or different did not appear to be correlated with the severity of the disability. Parent 6 whose child was moderately disabled said, "He is very different. He has a bad behavior (temper)." However, Parent 2 whose child had a strong behavioral component to his disability said, the following statement regarding her son's interaction with other extended family 
members, "They treat him the same way they treat their own kids." She continued to describe her son's relationship with the extended family by saying:

If they think that he is different, it is just a comparison they are doing between him, the way he acts, and the way other children his age or those smaller than him act. That is the only way they think he is different, which means they understand his problems better.

Parent 7, whose child had a moderate cognitive impairment, explained why she saw her daughter with disabilities as being different from a normally developing older brother. She stated:

Not exactly, but maybe because she is the baby. My daughter is different, I think, yes, maybe yes, because I don't want to leave her at home by herself. I don't want to leave her at anybody's house. I don't want anybody to harm her. In this way, I am really attached to her. We are attached to each other.

Parent 6 discussed the child by saying, "They all know that he is different from the other kids. That is why they treat him different."

\section{Home Behavior}

In this category, four parents gave detailed accounts of the child with disabilities' behavior at home. Many described the children as being constantly in motion. Parent 1 said, "Adam is very hyper. He is always high." Likewise, Parent 5 said, "No, she does things the opposite way. Even though I tell her to do something, she doesn't listen. She does it the opposite." Parents discussed the children's inability to follow directions as evidenced by the following comment from Parent 6:

He has a bad behavior (temper). You can tell him to do something, but if you don't watch him closely, take his hand to make sure he does it, he does not know how to do it. He will say "Ok Mom,", but he will not do it. I have another child who is younger than him and he is always telling him what to do. Any little conflict he has with the others, he starts banging on all the doors really loud. 


\section{Child's Attitude Versus Aptitude}

In this category six parents attributed their children's academic difficulties to reasons that made sense to them personally rather than those provided by formal evaluation results. Parents oftentimes attributed the children with disabilities' limited academic progress to their attitude rather than aptitude. Parent 1 had a child who exhibited behavioral difficulties and academic delays in school stated, "But he has the ability to do it. He is a little negligent. For me, it is because he is not able to control himself that is why he does not control himself.” In another instance, Parent 2, whose child had been categorized as learning disabled, described him in this manner, "I talk to the teacher. The teacher said that he does his work when it is explained to him. However, he does not try hard enough."

In the case of Parent 7, she felt that the program implementation procedures didn't challenge her daughter sufficiently. She said:

The program makes her lazy in a way. Sometimes, they ask her to do something, she says, "I don't feel like it." Believe me she can do 50\% more than what she does. Once she says no, you cannot force, but in Haiti, they would push her more.

The parents' comments demonstrated their ability to understand their children's specific disabilities and their perceptions of the impact the disability had on the children's academic performance. Parent 9, whose child was learning disabled, said:

My biggest problem with Isaac is I am asking myself why Isaac can't adapt himself like other children. Isaac does not want to try for his age. Every year, he is getting worse. Isaac does not perform. Everyday, I talk to him. I beat him up. I don't know what to do anymore. His father lives in New York. He has other children who are very intelligent. They do well in school.

Whose fault. Parents' responses indicated they had developed their own theories as to why their children were disabled. Some of the parents' conclusions were based on 
conversations with medical providers and others' were not. Parents identified their children's disabilities were the result of medical issues, something in the brain and not the school's fault. Parents described previous events and circumstances that they feel led to the children's current level of functioning. The reasons they provided allowed them to better understand why their children were disabled and how to deal with the disability.

Medical issues. Four parents identified medical events that resulted in their children being disabled. Parent 6 , whose child was born premature, said:

Since I know he is born premature. The doctors told he that he is considered to be in the family of disabled children, he is not totally disabled, but he is considered to be in that family of children. He goes to a special school since he was a baby. Every school he has attended, he has always been in a special program.

Parent 8 described the accident that resulted in the child having a disability in this manner:

He was in a coma for almost like 2 months. He was basically, actually, honestly he was pronounced dead after the accident. He got ejected from the car and he was pronounced dead, and then they put him in ICU on a respirator. He was in a coma. After 2 months, he came back. They had to teach him everything again, walking, talking, everything you name it. Everything had to be set back.

In the case of Parent 1, she identified seizures he suffered as a baby as the source of his current disability. She said, "Yes, he had three seizures." She went on to say that the milk given to him as a baby at the hospital also caused his current difficulties. She commented:

He developed a severe skin allergy to the milk. When I went to the doctor with him, he had an allergic reaction. They changed the milk. This is not his first skin or second or third skin on him right now. I said, perhaps this is what has affected him.

Something in the brain. Parents who did not have an understanding as to why their children were identified as being disabled attributed it to "something in the brain." 
Although they could not explain the specific root cause of the disability, they were satisfied with the explanation that it was caused by some type of brain dysfunction. Parents seemed to have formed this opinion from previous interactions with medical and educational professionals. As a result, three formed opinions regarding the cause of the disability. Parent 5 said, "They told me it's because she is slow, like her brain is not like the other kids." In the case of Parent 7, she sought treatment of the cognitive delays with doctors" visits and medication administration. She said, "I went to so many doctors, spent so much money. I put her on so many vitamins." Parent 9 said, "They did a lot of tests on him and did not find anything wrong with his brain.” These parents' responses demonstrated how they attempted to cope with their children's disabilities and the difficulties and challenged encountered as a result. They needed to understand why their children were disabled and used information from experts to formulate their explanations.

Not the school's fault. Although parents acknowledged that their children with disabilities necessitated special programs within the public school setting, four commented on their perceptions of the school's ability to meet its obligation to address the children's needs. Parent 3 said, "If the school gives him a first teacher, a second teacher, which means the school wants to help me." Parent 2 was in the process of seeking an alternative program for her child at the time of the interview. She had this to say about the school:

When I say that it is not rooted in the school, when I say there is an improvement, which is how he used to behave. There are some improvements, but there are others that are different. That is what I mean it does not come from the school. There are things that it is not that his parent wants him to do, but that is just the way he acts. 
Likewise, parent 8 commented on the lack of a support group for his son with the following comment:

I don't know if they have another program special for those kids like Henry is always saying, "Daddy, I would like to go and you know, be part of some young group." Whatever, you know and maybe I didn't do enough research around. Maybe, like I said, maybe those are probably my own fault. I didn't travel around to find out exactly what it's all about, but that is my fault.

\section{Core Concepts: Coping Mechanisms and Locus of Control}

After organizing the data into open codes, the categories were grouped under four themes. These themes evolved into two core concepts. The researcher analyzed the content of each category to determine the relevancy of each code tentatively included within it. The category of home school collaboration included comments made by parents on their communication with the school, how well they understood the process initiated by the school to place their children into special education, their evaluation of the program and its impact on their children, the nature of their interaction with the school, their assessment of their needs, the assistance needed from the school, and their parental involvement. Taken together, these codes gave insight into how parents interacted with the school. They provided insight into parents' perceptions of their role in the educational process and provided a blueprint for identifying their concerns, and priorities in the area of home school collaboration.

In the category of parental concerns, the parents commented on their financial struggles, their perceptions of "normal" behavior and/or development, their hopes and dreams for those children, and the resulting reactions they experienced when confronted with the fact these children had disabilities along with their concerns about the future, and the problems they faced in their everyday lives with these children. Taken together, 
these comments grouped together the stressors that dominated Haitian parents of children with disabilities and influenced their perceptions of those children. Parents' coping mechanisms were identified in their comments in these two themes. These two themes formed the core concept of the coping mechanisms used by parents to navigate through the myriad of issues encountered in dealing with children with disabilities.

The parents discussed the depth of their interactions with the school and the barriers and supports they needed to be more effective in their roles.

The third category looked at relationships/perceptions. It analyzed parents' relationships with various entities to determine how those relationships influenced their own perceptions of themselves and their ability to take control of their lives. This category comprised parents' relationships with God, how they perceived the children's interaction with siblings, the children's functioning within the larger community, the behaviors exhibited at home and how parents coped with those behaviors, and to what factors they attributed the children's limited academic progress. These comments provided insight into parents' perceptions of their own role in these children's lives and whether they felt empowered to effect change in those children's lives.

This last category addressed another component of parents' perceptions of their ability to control their lives and those of their children. It examined the comments parents made regarding the medical issues faced by their children, their perceptions of the causation of their children's disability, and who or what they felt was responsible. These comments allowed the researcher to hypothesize on the parents' disposition to address their children's difficulties and their ability to assist the children in compensating for their difficulties. These two categories identified parents' perceptions of their ability to 
control their current situations. The ability to feel in control of a situation is a prerequisite to active participation (Ariaza, 2000; Wathum-Ocama \& Rose, 2002).

\section{Coping Mechanisms}

The parents' ability to cope was evident in how they communicated with the school, the level of their involvement, their ability to understand the process, and how they reacted to the problems faced with these children whether academic or behavioral as well as what they viewed as issues of concerns for the future. Parents who exhibited strong coping mechanisms were active participants in their children's education and exhibited a higher level of parental involvement both at school and at home. Although these parents are from a culture where parental involvement is not an expectation (Stepick \& Stepick, 2003), they had internalized the demands in this new culture and made adjustments to their behavior to the benefit of their children. They were active participants in the educational program and had become acculturated. Therefore, they needed less support from the school. Parental coping mechanisms were also stronger in parents who identified fewer behavioral problems, fewer financial issues, more intensive and sustained interaction/communication with the school, and more positive concerns about the future. Although they realized that their children had difficulties, they attempted to cope with those challenges by becoming more active participants in the process.

\section{Locus of Control}

This conceptual theme revealed how parents perceived their challenges inherent in raising children with disabilities. The research identified internal locus of control as a method of perceiving one's problems or of reacting to situations. Internal locus of control 
dictated that parents look internally for solutions to their problems and an external locus of control caused them to look outside. Authors such as Swick and Hassell (1988), Fulgosi-Masnjak et al. (1996), Flouri (2006) and Hamlyn-Wright and Draghi-Lorenz (2007) suggested that locus of control influenced parents' perceptions and ultimately their interactions with schools. In this study, parents with an internal locus of control relied on their faith in God as a source of strength to empower them, sought to establish positive relationships with peers, and actively sought ways to engage the children more with family/friends and community. Parent 7 said, "She is a normal 15 year old. She handles everything. She knows all the keys [to the church where mother is a pastor]." When asked about her concerns for the future, Parent 10 said,

Well, I ask him what he wants to be, and he said he wants to be a preacher. So he wants to preach the word of God. Basically, whatever he wants to be, we will support him all the way.

Parents with an external locus of control believed it was the children's attitude rather to their aptitude that prevented them from being more successful academically. Parent 1 said this comment about her son's aptitude versus is attitude, "But, he has the ability to do it, He is a little negligent." Parent 5 said, "She will say that she can't do it, but really I know that she can do it.” Parent 4 said,

I think that the system can do much more for her because for all the time she has been at school, I have noticed what the system has done for her, how she is functioning, there is not a huge improvement. Now, she has very little time left before she leaves school, there is not much more they can do.

Some of the parents used their faith as a reason to give up and give in because it was not up to them. Parent 1 said, "If there is something to be done about his behavior, God will bring about this change in him. In the case of Parent 6 , she reacted to her child's behavior 
outbursts by distancing herself from it. She said, "He takes a lot of time to calm down. At those times, I stay in my room and close the door. He comes and bangs on my door." This parent blamed behavioral difficulties on the disability and did little to address them. Two parents stated children were disabled because of "something in the brain" and one said it was "not the school" or anyone else's fault. Parent 1 said,

The child demonstrates to you that he has sense, but he does not have any sense really. He demonstrates to you that he has all his sense about him, but according to what you see him doing, you can see he does not have any sense, really."

This parent also made the following comment regarding her son's need for medication.

They give me medications for him, but I am not sure exactly why they prescribe the medication. They were supposed to do a test in his brain. They never did it. They are always asking me questions about how he is.

These comments suggested that these parents, at times, were uncertain about the cause of their children's difficulties. Their comments also indicated limited understanding of how to begin to address the problems in meaningful ways. Therefore, they felt overwhelmed by the difficulties of dealing with a child with a disability and did not express confidence in being able to address these children's difficulties.

Locus of control also dictated how parents interacted with their children and schools to the benefit of those children. Those with an internal locus of control initiated and maintained contact with school personnel. Parent 8 said, "I ask for a meeting with them and then we sit down and talk about him." This parent also said, "I have several conferences with the teacher. Likewise, Parent 7 discussed being an advocate for her child with this comment, "I was there, I was always there. I kept checking so nothing happened." She added, "The teacher did not do a good job. I kept talking to the teacher, "How come she does not know her address, her phone number? These are things a child 
like her has to know. They saw a lot of me. I don't mind if you give her extra work, but she has to know these things." However, those will an external locus of discussed barriers that prevented them from being more involved. Parents in this study discussed how financial constraints prevented them from becoming more involved in their children education. Parent 4 and Parent 9 stated that they went to the school very infrequently because of their work schedules because they needed to work as single parents. These parents felt that their economic situations left them with limited choices regarding involvement. They were obliged to make finances a priority for the benefit of their families.

\section{Chapter Summary}

The categories that emerged from the data analysis answered the research question of a sample of Haitian parents' perceptions of their children with disabilities. Their perceptions were evident in the following categories: to be somebody, how parent reacts to child's problems, concerns about the future, problems, relationships with siblings, home behavior, interaction with family/friends/community (same \& different), and child's attitude versus aptitude. The first sub-question of parents' perceptions of the education their children were receiving was answered by the categories of: helpful program/improvement, the school can help, interaction/communication with the school, and not the school's fault. The second sub-question was parents' perceptions of their role in the education of their children with disabilities. The categories that answered it were: initial eligibility/understanding process, the school can help, I need help and parental involvement. The third sub-question concerned how these Haitian parents' perceptions of their child's disability influenced their relationship with the child and their involvement 
with the school. It was answered by: interaction with the school, parental involvement, the school can help, I need help, normal, to be somebody, how parent reacts to child's problems, problems, concerns about the future, problems, interactions with family/friends/community (same \& different), home behavior and child's attitude vs. aptitude. The fourth research sub-question was Haitian parents' perceptions of supports and barriers to their involvement in their children's education and was answered by: parental involvement, interaction/communication with the school and financial issues. Clearly, there was overlap in the responses that pertained to each research question (see Figure 2). This was due to the qualitative nature the research and the fact that parents had latitude in how they responded during the interview process. The results fleshed out the needs, concerns, and priorities of these Haitian parents of children with disabilities in the district where the study was conducted. 


\section{Research Question \\ Perceptions of Children}

- Parents want children to grow up to be somebody

- Parents are affected by children's problems

- Concerns about the future include independence and earning potential

- Parents encounter behavioral and academic difficulties with children

- Relationships with siblings are affected by disability

- Children with disabilities exhibit behavioral difficulties at home and learning problems at school

- Interactions with family/friends/community vary widely from close knit to distant

- Children's attitude vs. aptitude is the source of academic delays

\section{Sub question 1}

Perception of education

- Child is better now/has improved

- Need programs/services

- Interaction/communication hampered by language and time constraints

- School is not to blame for child's difficulties

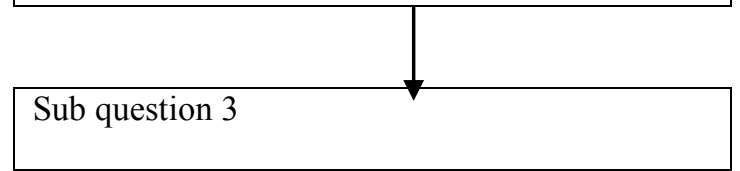

Perception of child/relationship with child/school

- Interaction hampered by language and time constraints

- Parental involvement is influenced by perception

- Parents need programs/services

- Parents need help with children financially, behaviorally, academically Parents see children as "normal"

- Parents want children to be somebody

- Parents' perceptions dictate how they react to problems

- Parents worry about the future

- Parents are stressed by problems

- Parents' perceptions influence interactions within family/community

- Children have problems adjusting

- Children's attitude vs. aptitude is the source of academic delays

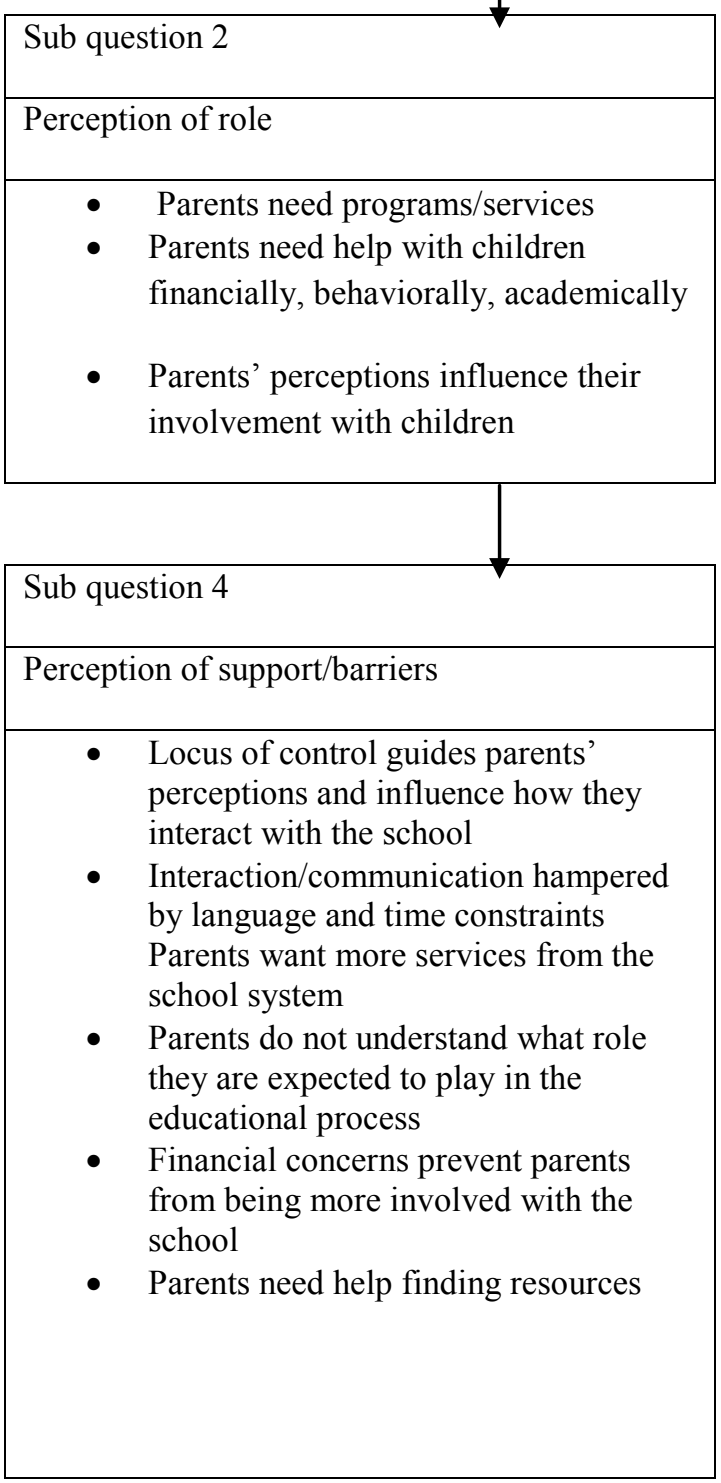

Figure 2. Grouping responses to research questions by categories. 


\section{CHAPTER V \\ DISCUSSION AND CONCLUSIONS}

The purpose of this study was to gain insight into a group of Haitian parents' perceptions of their children with disabilities by examining: (a) these Haitian parents' perceptions of the education their children were receiving; (b) their perceptions of their role in the educational process; (c) how their perceptions of the child affected their relationship with the child and the school; and (d) the barriers/supports they encountered in their involvement. This chapter presents a discussion of the research question and subquestions. The discussion will establish a link between previous research findings and parent responses obtained from the current research. Finally, it will discuss the limitations, make recommendations for current practice and provide suggestions for future research.

The two core concepts that emerged from the analysis of the data were coping mechanisms and locus of control. Evidence of parents' coping mechanisms were visible in their perceptions of their children, their perceptions of the educational program, their perceptions of their role, their relationship with the child and the school, and their perceptions of the supports and barriers to their involvement. Parents with more developed and sophisticated coping mechanisms had more positive perceptions of their children and were more capable of articulating the children's problems. An example is Parent 6 who said, "I go to the school when they call me, but you know my English is not so good. I don't find people to talk to ask anything of them." In contrast, Parent 7 said, I always talk to the teachers, sit in the classroom. I call and make sure they know me. I find 
out what is going on". Likewise, parent 8 said, 'Like I said, I keep track of everything he does. I will go to the school, talk with the teacher." Parent 6 also was less educated than Parents 7 and 8, a factor identified by Giles (1990) as hindering parental involvement in the American system. Parents 7 and 8 were also more aware of the parameters of the school's responsibilities. Furthermore, they had developed the skills and assertiveness to interact and communicate with the school to the benefit of their children. These parents appeared to have assimilated more fully to the norms and expectations of the American educational system and were, perhaps, more capable of confronting the challenge of raising a child with disabilities. Those with lower levels of coping mechanisms expected the school to provide more assistance, even when they could not clearly identify their own specific needs. For instance, Parent 4 said, "According to what I know, the school can help. They cannot help me in other ways. If they could help me in other ways, I would focus on it. However, I don't know about other programs." Parent 4 knew the school could help him, but was not specific about his needs. He had a lower level of engagement and communication with the school, perhaps because he lacked the skills to maneuver through the myriad of issues and policies in special education and their role in the process. Parent 6 said, “

There is a lady with a child similar to mine, her child is in many programs. She is not Haitian. She has a place for her child to go when he is 18 years old. I ask her how she found these programs. She tells me she will explain to me how to get them. She has not had the time yet.

Parent 4, whose child was in high school, was asked what would happen to the child after 22 years old. He said, "It would be a pleasure for me if the school could give me a resource to help me with this situation. They have not told me anything. If they did, that 
would be a big advantage for me and it would help me." Federal law under the Individuals with Disabilities Education Act (IDEA), Department of Education (2009), mandates transition be discussed prior to graduation from high school or the age of 22 for children with disabilities. This parent could not provide specific details as to whether this topic had been discussed during meetings. These parents seemed overwhelmed by the difficulties encountered with their children with disabilities. They also seemed to be encountering more problems currently and were more ambiguous about the future of their children and the children's ability to function within society.

The parents' coping mechanisms were also indicated by their responses to the children with disabilities. Those with an internal locus of control attempted to integrate them more with family and the larger community and seemed to have acculturated more fully into the educational system. They relied on faith and belief in God, but also were more active participants in their children's lives. Those with an external locus of control were more passive in their parental involvement. Parent 4 said, "I was expecting her to perform well in school, become a high level person, become a Ms. so and so like you, but based on what I see, she will not be able to accomplish that. I am obliged to accept it. It is done according to God's will. It is not done according to my will." Parent 1 said, "If I could find a special school for Adam, I would give thanks to God." Similarly, Parent 2 said, "If the school wants to help me in other ways, I would give thanks to God." They also relied on faith and belief in God, but used this belief as a reason to remain passive while waiting for divine intervention in their lives and that of their children. This latter group of parents also indicated they needed more services with less clarity on the specifics of the services needed. Finally, they were unsure about the future of their 
children because it was up to God and not up to them or their children. According to Swick and Hassell (1988), parents described their children's social competence in ways that were similar to their own social competence. Therefore, parents' perceptions in this arena was worthy for professionals to know. This information would assist not only in working with the children, but also in engaging the parents as well. Kalil and DeLeire (2002), conducted a study to examine the impact that financial difficulties had on locus of control. The authors identified that job loss and decreased finances led to long term effects on both children and adults. In the current study, several parents discussed having to work long hours and not having sufficient time to visit the school. Parent 4 stated he was a single parent and could not take time to go the school regularly. Parent 9 worked two jobs and stated she never went to the school. Both of these parents could have benefited from increased involvement with the school. They had limited educational backgrounds and lacked the skills to engage the children effectively in collaboration with the school. Therefore, their time constraints will adversely impact their children long term. These effects were manifested in children's limited academic progress, less consistent attendance and affected locus of control. Cogan and Oka (1992) found that parents who were able to manage their children had a more positive view of those children's continued intellectual functioning.

\section{Perceptions of Children}

The research conducted thus far within the field of special education with minority parents has determined that these parents oftentimes held views of their children that were different from the mainstream culture. That is, non-mainstream parents' 
conception of disability was not as prescriptive as those of professionals because they evaluated their children's functional levels based on the ability to function within the home environment. These perceptions could also be influenced by parents' acculturation and assimilation into the mainstream culture (Harry, 1992; Harry et al., 1995; Klein, 2008). This study is similar to previous findings regarding parents' conceptions of disability. Parent 4 said, "The problem is only her difficulty learning. She does everything else normal." This parent stated "I was expecting her to perform well in school, become a high level person." Parent 9 said, 'He did not know how to read before. That was his main problem, but now he reads a little." These two parents expected special education to resolve all their children's problems because Parent 4 expected the child to perform well in school after being placed in special education. The child had been categorized as having an intellectual disability. Likewise, Parent 9 had a child with learning disabilities, and she stated that the child's only problem was with reading. These parents stated that their children were independent at home and only had difficulties with reading and/or academic tasks. According to Harry (1992), the school system's description of what constituted as cognitively impaired tended to be more narrowly based and more descriptive than parents' view. Therefore, parents' perceptions of their children with disabilities were not correlated with either medical or educational conclusions identified in their educational records. Parents provided their own rationale of what constituted as a disability and how it was manifested in the individual child. Half of the parents in this study referred to their children as "normal" and considered them to be independent in the home. This creates a problem because the psychological evaluations and school records listed them as exhibiting mild to moderate cognitive impairments that 
resulted in significant delays in learning. The parents had a different perception of the criteria for determining disability.

\section{Perceptions of Education}

This research sub-question sought to identify parents' opinions of the education their children were receiving. Previous research indicated parents' perceptions of appropriate settings were different when they considered special education in general, and when they took into consideration the individual needs of their own child (Klein, 2008; Palmer et al., 2001). Parents did not feel confident or competent in challenging school personnel even when they did not agree with placement decisions (Harry, 1992). The parents in this study seldom disagreed with placement. Parent 4 said, "I would like for her to continue going to school I don't know how it can happen because I don't know a lot about the educational system here in this county or what can happen and if they can keep her. Anyway, my decision is for her to stay in school." However, he has not voiced this opinion to school personnel. Even when they did, they initially deferred to the "professionals" rather than voice their concerns. Parents in the current study also commented their children were making progress and that they were satisfied with the school program. However, they also commented that they expected more services and support from the school. When parents said they expected more from the school, their comments lacked specificity and it was not clear that they had voiced these desires to school personnel. Their comments demonstrated that they neither fully grasped the different layers of bureaucracy available at their disposal to address their concerns as parents of children with disabilities nor did they fully understand that they had a vital role to play in the process. 


\section{Perceptions of Role}

With this research question, the goal was to identify how these minority parents of children with disabilities internalized their role in their children's education and to what extent they fulfilled those roles. Research had concluded that culture played a substantial role in parental involvement and that parents from minority backgrounds often did not possess the knowledge necessary to be active participants in their children's education (Harry, 1992; Harry et al., 1995; Harry et al., 1999; McWayne \& Owsianik, 2004; Ouimette et al., 2004). This was also in line with the findings of this research. When asked if she was involved with her child's school, Parent 5 responded, "I don't know who to speak to. I don't know if it's the teacher or the principal." Some parents described aspects of the special education and placement process, but did not indicate that they fully understood its impact on their children's education and long term prognosis. They also failed to understand their expected role in the process. The parents lacked the knowledge to fully comprehend what special education entailed. Responses also indicated that a few parents lacked sufficient literacy skills to even begin to comprehend the process, much less their role in it. The research discussed socialization practices and acculturation and how they impacted parents' knowledge of parental roles and expectations in the larger society and their participation and their reliance on community values and beliefs (Harry, 2001; Hui-Chen \& Mason, 2008). The parents in this study may have been more susceptible to community influence even when those influences may have very little rational basis or are formed based on limited or faulty information. As a result, the perceptions they held were different from those of school personnel and negatively impacted their children's academic achievement. 
The parents' perceptions were guided by prior knowledge, education and cultural factors. Many parents referred to their children as "normal" irrespective of the difficulties these children were having or the educational diagnosis. The children were perceived to be normal by parents because they were seemingly normal and were capable of functioning within their home environments. Parent 4 said his daughter was normal because she did everything for herself independently at home and was able to cook and clean. Parent 9 said her son was normal, except that he could not learn how to read. These parents' perceptions of "normal" were based on prior knowledge of what constitutes as normal and also on their education and cultural knowledge. Within the Haitian culture, if one was independent and able to function within one's environment, then it was determined that this individual was normal and not a kokobe or someone with physical impairments. These parents' perceptions of these children were rooted in these cultural perceptions because their culture was their reference point. They had not acculturated sufficiently to the U.S. system of categorizing a person with disabilities which focuses on specific descriptions of capabilities and needs.

\section{Perceptions of Child and School}

In previous research on minority parents of children with disabilities, the authors gained insight into parental perceptions of the children from parents' interaction with the children. The parents' perceptions, in turn, dictated how they communicated with the school to the benefit of those children (Harry, 2001; Palmer et al., 2001). This research found parents would sometimes delegate responsibilities for supervision to siblings and would rely on siblings for the care of children with disabilities even if the siblings were younger. In a study conducted by Harry et al. (1995), it was determined that although 
parents were engaged initially in their children's education, this behavior faded over time and gave way to dissatisfaction and apathy. As children became older and more difficult to manage, parents' limited coping skills and needs were not addressed by the professionals instructing their children. Therefore, they disengaged from the school and became alienated. Parents in this study commented on the inability to communicate with school personnel and the limited resources available to them. They stated "I need help" or "the school can help" in requesting parental support groups and the availability of community resources to assist them with their children beyond school hours.

In this study, Parent 4 discussed how what he wished to have happen in the future for his daughter once she had matriculated beyond high school. However, he did not indicate that he had discussed transition services with school personnel. If that discussion had taken place at a meeting, it possible he did not fully understand the topic was being discussed. He did not understand his expected role as parent was to inquire of school representatives what would happen to his child once she exited the public school program. He acknowledged to the researcher during the interview that he did not understand how the U.S. educational system functioned and whether high school and college were governed by the same rules. He did not know that he had the right to ask and be given answers. As a result, he was not specific about the next step in his child's future. He had no transitional plan in place for her once she finished school and did not know how or where to get assistance to resolve this impending issue. Likewise, parent 9 stated they told her at the initial placement meeting that her son was being placed in an exceptional program for children with disabilities, but they did not tell her for how long. This parent's comment indicated that she likened placement into special education to 
taking a medication prescribed by a medical provider. The patient is told what medication will cure the illness and how long it is to be taken. Both of these comments demonstrated that these parents did not fully understand their expected role in the special education process. They were unable to function as advocates for their children. Again this points to cultural factors as well because, in Haitian culture, parents were not expected to question school personnel or to engage in their children's education to such a degree. Special education services tailored to address children's needs were not established as part of the general education services.

\section{Perceptions of Support /Barriers}

In previous research, some of the barriers parents identified as hindering their involvement in their children's education included limited education, lack of knowledge, limited skills and resources, limited English language proficiency, and time constraints (Al-Hassan \& Gardner 2002; Barton et al., 2004; Musti-Rao \& Cartledge, 2002; Smrekar, \& Cohen-Vogel, 2001; Turney \& Kao, 2009; Wherry, 2004a). These were also factors that parents identified as influencing their ability to participate in their children's education in this research. Parents mentioned having to work multiple jobs and having insufficient time to come to school, being unable to communicate effectively with school personnel in the absence of interpreters and lacking knowledge of the resources available to them. Although, these findings were supported by previous research on the barriers that have negatively influenced parental involvement within minority groups, they continue to exist in this research as well because they have not been sufficiently addressed. Therefore, educators need to analyze parental involvement from the parents' perspective so that they can formulate ways to engage these parents effectively in their 
children's education. Any services implemented must be the result of a thorough analysis of individual parental needs.

\section{Supports}

In traditional Haitian culture, public education is not as accessible as it is here in the U.S. In fact, in Haiti private education is more prevalent and of higher quality than public education. As a result the literacy rate is low. As the poorest country in the Western Hemisphere, Haitians live on less than $\$ 2.00$ a day. Therefore, schooling is not a priority when compared to food, shelter and clothing. For those few who can access the miniscule number of slots available in public schools, textbooks, and other necessary materials are to be provided by parents (Wikipedia, 2010). In this context, parents interviewed for this study identified the school as being a partner. The majority stated that the programs available to their children had been beneficial to them. Parent 1, said the program was helping her. The access to free public education with textbooks provided was beyond their expectations. As a result, parents expected the school to provide services beyond what was already available. Parent 2 said she always told school personnel her problems so that they could help. Parent 4, said the more school could help, the happier he would be. Parent 10 said she wanted her son to be in programs because she did not have time to help him. These parents expected the school to provide services beyond what was already at their disposal. They saw the school as a partner that had unlimited resources and unlimited restrictions.

\section{Barriers}

Among the barriers identified by parents in this study, language was the most prevalent. Wathum-Ocama and Rose (2002) found that language was one of the barriers 
preventing parents from participating in their children's education. This was the case in this study as well. Parents 4 and 6 discussed the difficulties they had communicating with school personnel even when they could attend meetings. However, the presence of a translator at meetings seemed to be an incidental event. The parents had translation only if one of the individuals scheduled to be at the meeting happened to speak the parents' language. Otherwise, no concerted effort was made to provide translation in the home language during meetings. The parents also failed to request the services of a translator and instead nodded agreement to all items discussed during meetings, even when they understood very little of what was occurring in the meetings. Again, this pointed to cultural factors. Haitians tended to be reluctant to voice their needs. They felt it was not their place to make any requests or demands of school personnel who were regarded as people professionals and not to be questioned.

Another barrier that parents identified as limiting them was finances. Parent 1 discussed her limited resources and having to work nights to provide for the family. Parents 4, 6, and 9 all described long work hours that interfered with their participation in school meetings. In these instances, parents felt that although they wanted to interact more with school personnel, their economic situations restricted them. Also, 9 of the 10 parents interviewed for this study were single. Therefore, they were the only income earner in the family. In all instances, parents had more than one child in the home. This situation no doubt added stress to the family because parents had an obligation to support the family and this was prioritized above parental involvement and participation in school activities. 


\section{Limitations}

Findings from the current study revealed that these parents of Haitian children with disabilities had various concerns that have influenced their perceptions of these children as well as their perceptions of the education the children were receiving. However, these findings should be taken with caution in light of the size of the sample. A study with a larger group of parents might yield different results. The age range of the children participants might also have affected these results. Children had been in special education from 2 to 10 years or more based on their grade level at the time of placement and the grade at the time of the study. For example, the parents who agreed to participate had children in different grade levels from elementary to senior high school at the time of the study. Therefore, a more homogeneous group of parents may have had different experiences, hence different comments. Finally, this study was conducted with only one interview. Another study with several encounters may also lead to different results.

Another limitation of this was study was the varied backgrounds of the parents. They had different levels of educational backgrounds from elementary to post graduate. This undoubtedly affected their assimilation and acculturation into the U.S. educational system. A group of parents with similar educational backgrounds may have provided different responses. Additional limitations were English language proficiency, socioeconomic status and careers. These different variables undoubtedly influenced parent concerns and priorities. This study does not take into account the correlation among the concerns they had identified, their prior knowledge, their experiences both here in the U.S. and abroad and the ability to participate effectively in their children's education. 
These factors taken wholly or in part may have impacted their perceptions of the children as well as their expectations of the school system.

This study involved Haitian parents of children with disabilities within in a large South Florida urban school district. The group interviewed was Haitian parents of students already participating in a program for students with disabilities. Parents of children exhibiting academic difficulties, but not yet identified as having a disability might hold different opinions than those included in this study. These parents would have been at a different point in the process and both their involvement and their perceptions might vary as a consequence. Additionally, the first set of letters sent out to parents requesting their participation in the study resulted in no responses. It was necessary to contact parents individually by phone to secure verbal consent prior to a second letter being sent to secure parent agreement to participate in the study. This study did not analyze the rationale behind the reluctance to respond to the initial request to participate nor did it explore parental disagreement to be interviewed.

The district where the study was conducted was very large. However, ethnic groups tended to congregate in certain areas more than others. The district granted permission for the researcher to access schools in close proximity to one other. This also may have been a contributing factor in the results since all the parents were within the same geographic area. This was coupled with the fact that parents were self selected. Interviewing parents from the entire spectrum of the district or different participant selection methods might also yield different results. 


\section{Implications for Current Practice}

Information regarding these Haitian parents can be useful to professionals as they seek to implement programs and services to address the needs of Haitian parents and their children. Professionals need to understand parents' viewpoints, their backgrounds, beliefs and value system as well as how these factors influence parental behavior (Diamond et al., 2004). Professionals also may not be culturally competent and may have negative perceptions of racial/ethnic parents (Ariaza, 2004). The rationale for this pattern of behavior stems from the fact that the mainstream culture fails to acknowledge that people of diverse racial/ethnic backgrounds have beliefs and practices that are different from the dominant culture. It also originates in the fact that being from a non-mainstream background is considered the first layer and that the disability adds a second layer. Professionals tend to treat these minority groups as if their needs and/or concerns are not important because the mainstream culture has not even begun to address the primary layer, which is that minorities have different needs, concerns, and priorities (Harry, 2001; Harry 2008).

It is recommended that this school system take more of an active role in engaging parents and assessing their needs so that they can be addressed. This is especially critical for parents who are limited English proficient. There seemed to be no systematic procedures in place to address the needs of these parents. That is unfortunate because it indicates a disregard for federal mandate and the value of parent participation. As a result, professionals may not extend to parents the respect, deference and opportunity to provide input necessary so that they can participate more actively in their children's education. Involvement is necessary from the parents or guardians of the children being 
served and is a precursor to appropriate adjustments that can be made to incorporate the needs of not only the students, but also parents as well in the process of educating children.

The findings of study are similar to previous findings by Stepick and Stepick (2003), which concluded that Haitians in South Florida emigrated there for several reasons, not the least of which was the educational opportunities for themselves and their children. Stepick and Stepick (2003) overwhelmingly found Haitians held the belief that education was the primary vehicle through which they could overcome their current difficulties and achieve a better future. This study advanced the body of knowledge by obtaining similar results with a specific sub-group of Haitian parents. Additionally, it provided insight into these parents' coping mechanisms and locus of control. Their perceptions greatly influenced parents' thoughts and behaviors when they realized that their children with disabilities might perhaps fall short of the promises they held out for those children as descendants of immigrants who had been expected to become success makers in this country.

In light of the documented need for increased parental involvement, especially with regard to CLD groups with disabilities, this district needs to implement more professional development to assist professionals in becoming more culturally competent. It has been suggested that professional preparation programs should embed cultural competence in their programs because teachers' knowledge and attitudes can increase or decrease parental involvement (Caspe, 2003, Chavkin, 2005; DePlanty, Coulter-Kern, \& Duchane, 2007; Deslandes, Fournier \& Morin, 2008; Knight, 2008; Sohn \& Wang, 2006, Souto-Manning \& Swick, 2006; Quintanar \& Warren, 2008). Professionals must take an 
active role in engaging parents and preparation programs need to give them the knowledge and awareness to demonstrate this skill. After entering the field, professionals need to be supported through ongoing professional development. The ability to engage parents needs to become part of the annual assessment process. It is one method of increasing accountability and translating learned behavior into practice.

Professionals also need to take into consideration parents' coping mechanisms and identify their locus of control in order to evaluate their needs more effectively so as to provide the support necessary to cultivate involvement. Klein (2008) found that immigrant parents who were able to engage in their own pursuit of knowledge independent of the school system participated more effectively in their children's education as a result of the knowledge gained. The parents in Klein's study (2008) had developed the cultural competence to identify the specific skills necessary to function more effectively within the American educational system. Furthermore, they had taken steps to acquire and demonstrate those skills. As a result, they had been socialized to the norms of the mainstream culture and this was indicative of more developed coping mechanisms. However, those with less developed coping mechanisms had not fared as well because of their limited ability to acquire the skills necessary to participate in their children's education. Professionals need to conduct a needs assessment of parental skill level and ability as a precursor to implementing any program activity aimed at increasing parental involvement and home school collaboration.

Professionals also need to understand parental locus of control. Although it may be difficult for professionals to change parental locus of control from external to internal, it is important to understand parents' locus of control. This is a beginning step in 
identifying how parents formed their perceptions and for professionals to gain insight into the influences on parental behavior and involvement. Harry et al. (1999) identified nuances of parental involvement within groups. Therefore, goals for increasing parental involvement must be individualized based on the specific needs of each individual family. This practice can increase the success rate of intervention aimed at increasing parental involvement, which is not only vital, but a federal mandate.

Findings from this study revealed that all parents except one were born in Haiti. Although they had similar needs, concerns and priorities, there were also nuances within the group. Arzubiaga et al (200) found this phenomenon in a study conducted with CLD learners. The nuances in this study were evidenced by Parent 7, 8 and 10 who had the highest levels of education. As such, they had also acculturated sufficiently to learn the parental expectations in the American educational system. They spoke about visiting classes, requesting meetings with school personnel and critically analyzing the instruction, curriculum, and home learning respectively. The role of parents in Haiti was very different from the U.S. Yet, they had assimilated and were able to demonstrate their newly learned skills to the benefit of their children. Professionals seeking to duplicate their success with a broader range of Haitian parents of children with disabilities will need to take into account how they acquired those skills. Professionals can then use this knowledge to provide skill building training for other less highly educated Haitian parents of children with disabilities.

Additionally, language was found to be a significant factor in these parents' level of engagement. More than $50 \%$ of the interviews were conducted in the home language because parents felt more comfortable and were able to express themselves more easily in 
Haitian-Creole. Yet, the school system where the study was conducted did not have any special measures in place to routinely provide translations to parents of children with disabilities at meetings. IDEA requires that notice of meetings be given to parents in a language they understand. A review of district school records revealed that there was a place provided in the written notices sent home for parents to request a translator. The review also indicated that very few used this option upon returning the form. Harry (1992) a found that parents felt that schools were not welcoming and that parents deferred decision-making to school personnel even when then did not agree with those decisions. She also found that parents were overwhelmed by all the written communication they received from school. Additionally, Harry (1995) found that the special education system was inflexible to parents' needs and was often times rigid. Her findings were supported by this study in part because parents reported inability to communicate with school personnel. This lack of communication resulted in miscommunication and formulations of ideas and opinions on the side of both parents and school personnel about each other that were likely erroneous.

It is suggested that school personnel seek meaningful of ways to engage Haitian parents of children with disabilities. It is important to identify the specific needs of parents on an individual basis prior to initiating the provision of services. Darch, Miso and Shippen (2004) suggested a prescriptive approach to parental involvement where professionals recruited parents, developed materials based on their needs, provided training and sought ways to sustain their engagement. Harry, Rueda and Kalyanpur (1999) likewise, suggested professionals needed to understand a particular group's ethnic and cultural beliefs and that goals should be framed around that particular group's needs. 
Finally, Gilles (1990) wrote specifically about Haitian parents and suggested that professionals seek to understand why parents cannot participate with schools. This process would be most beneficial to address the needs of Haitian parents of children with disabilities and engage them in the educational process for their children. In the end, the children and parents would both experience a more positive interaction within the U.S. educational system. Professionals would also gain new understanding of the particular needs, concerns, and challenges of Haitian parents of children with disabilities.

\section{Recommendations for Future Research}

Researchers have found that to improve teacher parent interactions, teachers should have a working understanding of parents' participation in their children's education. Since parental input is so crucial to the process, parents' perception of their role is a starting point to understanding, and therefore, interpreting parents' behaviors. The parents in the present study were also dissatisfied with the school's failure to communicate with them during the evaluation and placement of their children with disabilities. The parents stated that they were only given the results at the initial placement meeting. Additional research can address how schools can better educate parents so that they are made to feel they are part of the process.

Only one interview was conducted with each parent during the course of this study. A study with several encounters may also yield different responses and provide more in-depth information on both the parents and children. Although, the researcher had spoken to all the parents on the phone prior to the face to face interviews, this study involved only one direct encounter. Also, the majority of the parents were single. Future research can attempt to isolate parents' marital status as a controlled variable in the study. 
Another aspect of parents' responses worth mentioning was their relationship with God. More than half of the parents described a strong relationship with God and regular attendance at church. This relationship with God was both a coping mechanism and a crutch for parents who were oftentimes overwhelmed by the pressures of dealing with their children with disabilities. Those who used it as a coping mechanisms actively participated in their children's educational, social, and emotional development and used it as a source of strength and a coping mechanism. Those who used it as a crutch failed to be active participants in their children's lives and expected their faith to resolve all problems encountered with the children. This research identified the existence of a relationship between religion and parents of children with disabilities. The nature of this relationship, its causation or resulting impact on the parents is not clearly evident in this research. This relationship needs to be analyzed further. Future research can address what role religion played in these parents' lives, whether the impact can be generalized across different religions and how religion influenced parental perceptions of their children. Additional research can also examine the impact of religion on parental practices and their involvement in their children's education.

In this study, the research was conducted by someone of the same background as the parents. This facilitated communication with the participants and the establishment of rapport. Thus far, research on parents of children with disabilities has not been widespread. People of ethnic and racial minorities and CLD groups have specific identities, needs and challenges as outlined in the various research studies previously mentioned. The prerequisite to conducting research within a specific group is an interest in and/or specific knowledge regarding the specific sub-group within which the research 
is conducted. That is the vehicle through which additional knowledge is gained regarding specific CLD populations and/or sub-groups. It suggested that more researchers undertake studies with minority CLD groups, especially those with disabilities. Such studies will add to the body of knowledge and increase awareness of their priorities and needs and whether these are unique in nature.

The presence of a disability has been found to add new challenges and pressures on family. No doubt, being an immigrant exacerbates those challenges. Research on minority groups and the nature of their participation in the education of their children with disabilities has been done on different immigrant groups. The demographic changes in the U.S. indicate that over time, the number of people from racial/ethnic minority groups in this country will continue to increase. It is suggested that future research continue with minority groups in this county. Researchers need to identify the role of such components as cultural competence and socialization as factors contributing to increased parental involvement.

This study added to the body of knowledge because it was done with Haitian parents of children with disabilities, an ethnic minority in the U.S. and the third largest ethnic group in the district where the study was conducted. Insight into those parents' perceptions will enlighten professionals in collaborating with them with the result of providing their children with a free, appropriate public school education as mandated by law. The study will provide insight that will assist professionals in formulating programs to meet the needs of parents as they strive to increase parental involvement. 


\section{Discussion Summary}

This chapter discussed the findings of the research and elaborated on the conclusions that can be drawn from the results obtained. It explored the two core concepts of coping mechanisms and locus of control and discussed how they impacted Haitian parents of children with disabilities' ability to come to terms with their children's limitations. It outlined similarities between this study and previous findings within the research question and sub-questions which concerned Haitian parents': (a) perceptions of children, (b) perceptions of education, (c) perceptions of their role, (d) perceptions of the child and the school, and (e) perceptions of support and barriers to their involvement. Additionally, it outlined the limitations of the current research and its implications for current practice. Finally, it made recommendations for future research within the field. 


\section{REFERENCES}

Al-Hassan, S., \& Gardner, R. (2002). Involving immigrant parents of students with disabilities in the educational process. Teaching Exceptional Children, 34(5), 5258 .

Ariza, E. (2000). Actions speak louder than words-or do they? Debunking the myth of apathetic immigrant parents in education. Contemporary Education, 71(3), 36-38.

Ariza, E. (2002). Cultural considerations: immigrant parent involvement. Kappa Delta Pi Record, 38(3), 134-137.

Ariaza, G. (2004). Making changes that stay made: school reform and community involvement, The University of North Carolina Press, 87(4), 10-24.

Arzubiaga, A., Artiles, A., King, K., Harris-Murri, N. (2008). Beyond research on cultural minorities: Challenges and implications of research as situated cultural practice, Exceptional Children, 74(3), 309-327.

Barton, A.C, Drake, C., Perez, J.G., St. Louis, K. \& George, M. (2004). Ecologies of parental engagement in urban education. Educational Researcher, 33(4), 3-12.

Bogdan, R. C. \& Biklen, S.K. (2007). Qualitative research for education: An introduction to theories and methods (3rd ed.). Boston: Pearson Education.

Cartledge, G., \& Kourea, L. (2008). Culturally responsive classrooms for culturally diverse students with and at risk for disabilities. Exceptional Children, 74(3), 351371.

Caspe, M. (2003). How teachers come to understand families. Retrieved February 3, 2005, from

http://www.gse.harvard.edu/hfrp/projects/fine/resources/digest/hon_kong.html

Chavkin, N. F. (2005). Strategies for preparing educators to enhance the involvement of diverse families I n their children's education. Multicultural Education, 13(3), 1620.

Chen, J. L. (2005). Perceived academic support from parents, teachers and peers: Relation to Hong-Kong adolescents' academic behavior and achievement. Retrieved March 24, 2006, from http://www.gse.harvard.edu/hfrp/projects/fine/resources/digest/hon_kong.html 
Chrispeels, J., \& Gonzalez, M. (2004). Do educational programs increase parents' practice at home? Factors influencing Latino parent involvement. Retrieved March 24, 2006, from http://www.gse.harvard.edu/hfrp/projects/fine/resources/digest/latino.html

Cogan, L. S., \& Oka, E. R. (1992, April). Maternal beliefs and children's learning: A developmental perspective. Paper presented at the Annual Meeting of the American Educational Research Association, San Francisco, CA.

Cordry, S., \& Wilson, J. (2004). Parents as first teacher. Education, 125(1), 56-63.

Creswell, J. W. (2003). Research design: Qualitative, quantitative and mixed methods (2nd ed.). Thousand Oaks, CA: Sage.

Darch, C., Miao, Y., \& Shippen, P. (2004). A model for involving parents of children with learning and behavior problems in the schools. Preventing School Failure, 48(3), 24-34.

Department of Education, Chapter III Office of Special Education and Rehabilitative Services, Retrieved October 5, 2009 from http://www.access.gpo.gov/nara/cfr/waisidx_02/34cfr300_02.html

DePlanty, J., Coulter-Kern, R., \& Duchane, K. (2007). Perceptions of parent involvement in academic achievement. The Journal of Educational Research, 100(6), 361-368.

Deslandes, R., Founier, H., \& Morin, L. (2008). Evaluation of a school, family, and community partnerships program for preservice teachers in Quebec Canada. Journal of Educational Thought, 42(1), 27-51.

Diamond, J. B., Wang, L., \& Gomez, W. K. (2004). African American and ChineseAmerican parent involvement: the importance of race, class, and culture. Retrieved February 3, 2005, from http://www.gse.harvard.edu/hfrp/projects/fine/resources/digest/race.html

Ediger, M. (2008). Psychology of parental involvement in reading. Reading Improvement, 45(1), 46-52.

Epstein, J.L. (2008). Improving family and community involvement in secondary schools. Education Digest, 73(6), 9-12.

Flouri, E. (2006). Parental interest in children's education, children's self-esteem and locus of control, and later educational attainment: Twenty-six year follow-up of the 1970 British Birth Cohort. Journal of Educational Psychology, 76, 41-55. 
Fulgosi-Masnjak, R., Gustovic-Ercegovac, A. \& Igric, L. (1996, July). The connection between some dimensions of perceived personal competence and permanent low intensity stress in parents of children with intellectual disabilities. Paper presented at the Annual world Congress of the International Association for the Scientific Study of Intellectual Disabilities, 10th Helsinki, Finland.

Giles, H.C. (1990). Counseling Haitian students and their families: issues and interventions. Journal of Counseling \& Development, 68 (1), 317-320.

Ginsberg, R., \& Herman-Ginsberg, (2005). Accomplished teachers and their interactions with parents: a comparative analysis of strategies and techniques. Retrieved March 24, 2006, from http://www.gse.harvard.edu/hfrp/projects/fine/resources/digest/accomplished.html

Griego Jones, T. (2002). Incorporating Latino parents' perceptions into teacher preparation. Retrieved March 24, 2006, from http://www.gse.harvard.edu/hfrp/projects/fine/resources/digest/accomplished.html

Halsey, P. A. (2004). Nurturing parental involvement: two middle level teachers share their secrets. The Clearing House, 77(4), 135-137.

Hamlyn-Wright, S., Draghi-Lorenz, R. \& Ellis, J. (2007). Locus of control fails to mediate between stress and anxiety and depression in parents of children with a developmental disorder. Autism, 11, 489-501.

Harry, B. (1992a). An ethnographic study of cross-cultural communication with Puerto Rican American families in the special education system. American Educational Research Journal, 29(3), 471-494.

Harry, B. (1992b). Making sense of disability: Low-income, Puerto Rican parents' theories of the problem. Exceptional Children, 59(1), 27-40.

Harry, B. (1996). These families, those families: the impact of researcher identities on the research act. Exceptional Children, 62(4), 292-300.

Harry, B. (2001). Trends and issues in serving culturally diverse families of children with disabilities. The Journal of Special Education, 36(3), 131-138.

Harry, B. (2008). Collaboration with culturally and linguistically diverse families: ideal versus reality. Exceptional Children, 74(3), 372-388.

Harry, B., Allen, N., \& McClaughlin, M. (1995). Communication versus compliance: African-American parents' involvement in special education. Exceptional Children, 61(4), 364-377. 
Harry, B., \& Klingner, J. (2006). Why are so many minority students in special education? Understanding race \& disability in schools. New York: Teachers College Press.

Harry, B., Rueda, R., \& Kalyanpur, M. (1999). Cultural reciprocity in sociocultural perspective: Adapting the normalization principle for family collaboration. Exceptional Children, 66(1), 123-136.

Hoover-Dempsey, K. V., Bassler, O., \& Brissie, J. S. (1992). Explorations in parentschool relations. Journal of Educational Research, 85(5), 287-294.

Hoover-Dempsey, K. V., Bassler, O., \& Burrow, R. (1995). Parents' reported involvement in students' homework: strategies and practices. The Elementary School Journal, 95(5), 435-450.

Hoover-Dempsey, K. V., Battiato, A.C., Walker, J. M. Reed, R.P., Dejong, J. M. \& Jones, K. P. (2001). Parental involvement in homework. Educational Psychologist, 36,195-210.

Hoover-Dempsey, K. V., \& Sandler, H. (1995). Parental involvement in children's education: Why does it make a difference? Teacher's College Record, 97(2), 310331.

Hoover-Dempsey, K. V., \& Sandler, H. (1997). Why do parents become involved in their children's education? Review of Educational Research, 67(1), 3-42.

Hoover-Dempsey, K. V., Walker, J. M., Jones, K., \& Reed, R. P. (2002). Teachers involving parents (TIP): Results of an in-service teacher education program for enhancing parental involvement. Teaching and Teacher Education, 18(7), 1-25.

Hui-Chen, G., \& Mason, K. (2008). Motivations of parental involvement in children's learning: Voices from urban African American families and preschooler. Multicultural Education, 15(3), 20-7.

Hurtig, J. (2004). Parents write their worlds: A parental involvement program bridging urban schools and families. Retrieved March 26, 2006, from http://www.gse.harvard.edu/hfrp/projects/fine/resources/digest/urban.html

Jeynes, W. (2005). Parental involvement and student achievement: A meta-analysis. Retrieved October 9, 2006, from http://www.gse.harvard.edu/hfrp/projects/fine/resources/digest/reading.html

Johnson, J. O., Kominski, R. Smith, K., \& Tillman, P. (2005). Changes in the lives of U.S. children 1990-2000U.S. Census Bureau. Retrieved March 19, 2009, from http://www.census.gov/population/www/documentation/twps0078/twps0078.html 
Kato-Otani, E. (2004). Story time: Mothers' reading practices in Japan and the U.S. Retrieved March 24, 2006, from http://www.gse.harvard.edu/hrrp/projects/fine/resources/digest/reading.html

Klein, A. (2008). From Mao to Memphis: Chinese immigrant fathers' involvement with their children's education. The School Community Journal, 18(2), 91-117.

Knight, C. (2008). Celebrating immigrant learners. Democracy \& Education, 17 (3), 5457.

Kominski, R., Jamieson, \& Martinez, G. (2001). U.S. Census Bureau. Retrieved March 20, 2009 from http://www.census.gov/population/www/documentation/twps0052/twps0052.html

Lunts, E. (2004). Math teachers' use of class websites to support parent involvement. Retrieved February 3, 2005, from http://www.gse.harvard.edu/hfrp/projects/fine/resources/digest/reading.html

Lynch, S. Hurford, D. P. \& Cole, A. (2002. Parental enabling attitudes and locus of control of at-risk and honors students. Adolescence, 37(147), 527-549.

McWayne, C., \& Owsianik, M. (2004). Parent involvement and the social and academic competencies of urban kindergarten children. Retrieved March 24, 2006, from http://www.gse.harvard.edu/hfrp/projects/fine/resources/digest/boston.html

Miller, E.R. (2001). U.S. Census Bureau. Retrieved March 20, 2009, from http://www.census.gov/population/www/documentation/twps0054/twps0054.html

Murray, M., \& Mandell, C. (2004). Evaluation of a family-centered early childhood special education preservice model by program graduates. Top Early Childhood Special Education, 24(4), 238-249.

Musti-Rao, S., \& Cartledge, G. (2004). Making home an advantage in the prevention of reading failure: strategies for collaborating with parents in urban schools. Preventing School Failure, 48(4), 15-21.

Nagel, N.G., \& Guest, S. (2007). Helping family-school-community partnerships thrive: a conversation with Joyce L. Epstein. Democracy \& Education, 16 (4), 2-5.

Occasional Papers Series Dialogues. (1982). Haitians in Miami: An assessment of their background and potential. Miami, FL: Latin American and Caribbean Center, Florida International University. 
Ouimette, M., Feldman, J., \& Tung, R. (2004). Engaging parents in an urban public high school: A case study of Boston arts academy. Retrieved February 3, 2005, from http://www.gse.harvard.edu/hfrp/projects/fine/resources/digest/boston.html

Palmer, D., Fuller, K., Arora T., \& Nelson, M. (2001). Taking sides: Parent view on inclusion for their children with severe disabilities. Exceptional Children, 67(4), $467-484$.

Quintanar, A.P. \& Warren, S.R. (2008). Listening to the voices of Latino parent volunteers. Kappa Delta Pi Record, 44(3), 119-123.

Redding, S., Langdon, J., Meyer, J., \& Sheley, P. (2004). The effects of comprehensive parent engagement on student learning outcomes. Retrieved February 3, 2005, from http://www.gse.harvard.edu/hfrp/projects/fine/resources/digest/boston.html

Schmidley, A. D., \& Robinson, J.G. (2003). Measuring the foreign-born population in the United States with the current population survey: 1994-2002. U.S. Census Bureau. Retrieved March 19. 2009, from http://www.census.gov/population/www/documentation/twps0073/twps0073.html

Seidman, Irvin (2006). Interviewing as qualitative Research: A guide for researchers in education and the social sciences (3rd ed). New York:Teachers College Press.

Sensiper, S. (1999). Generating family-school partnerships through social marketing. Retrieved March 24, 2006, from http://www.gse.harvard.edu/hfrp/projects/fine/resources/digest/boston.html

Sheridan, S., Clarke, D., Marti, D., Burt, J., \& Rohlk, A. (2005). Conjoint behavioral consultation: A model to facilitate meaningful partnerships for families and schools. Retrieved April 14 2005, from http://www.gse.harvard.edu/hfrp/projects/fine/resources/digest/boston.html

Skiba, S., Simmons, A., Ritter, S., Gibb, A., Rausch, K., Cuadrado, J., \& Chung, C. (2008). Achieving equity in special education: History, status, and current challenges. Exceptional Children, 74(3), 264-288.

Smith, A. S., Ahmed, B., \& Sink, L. (2000). An analysis of state and county population changes by characteristics: 1990-1999. U.S. Census Bureau. Retrieved March 20, 2009, from

http://www.census.gov/population/www/documentation/twps0045/twps0045.html

Smrekar, C. \& Cohen-Vogel, L. (2001). The voices of parents: Rethinking the intersection of family and school. Peabody Journal of Education, 76 (2), 75-100. 
Sohn, S. \& Wang, X. C. (2006). Immigrant parents' involvement in American schools: Perspectives from Korean mothers. Early Childhood Education Journal, 34(2), 125-132.

Souto-Manning, M., \& Swick, K. (2006). Teachers' beliefs about parent and family involvement: Rethinking our family involvement paradigm. Early Childhood Education Journal, 34(2), 187-193.

Stepick, A. (1998). The new immigrant series pride against prejudice: Haitians in the United States. Needham Heights, MA: Allyn \& Bacon.

Stepick, A., \& Stepick, C.D, (2003). Immigration, identity, intergenerational relations, and academic orientation. American arrivals: Anthropology engages the new immigration. Santa Fe, NM: School of American Research Advanced Seminar Series, School of American Research Press.

Strauss, A., \& Corbin, J. (1998). Basics of qualitative research techniques and procedures for developing grounded theory ( $2 \mathrm{nd}$ ed.). Thousand Oaks, CA: Sage.

Swick, K. J. \& Hassell, T. (1988). Parental efficacy and the development of social competence in young children. U.S. Department of Education Office of Educational Research and Improvement, 1-15.

Trent, S., Kea, C., \& Oh, K. (2008). Preparing preservice educators for cultural diversity: how far have we come? Exceptional Children, 74(3), 328-350.

Turney, K. \& Kao, G. (2009). Barriers to school involvement: Are immigrant parents disadvantaged? The Journal of Educational Research, 102(4), 257-271.

U.S. Census Bureau, (2000). American FactFinder. Retrieved March 19, 2009, from http://factfinder.census.gov/servlet.GCTTable?_bm=y\&-geo_id=04000US12\&ds_name $=$

U.S. Census Bureau (2009). State \& County QuickFacts. Retrieved March 20, 2009 from http://quickfacts.census.gov/qfd/states/12/1245000.html

Wathum-Ocama, J.C., \& Rose, S. (2002). Hmong immigrants' view on the education of their deaf and hard of hearing children, American Annals of the Deaf, 147(3), 4453.

Wherry, J. (2004a). Walk a mile in parents' shoes. Principal, 82(3), 6.

Wherry, J. (2004b). Two perspectives on parent involvement. Principal, 83(5), 6.

Wherry, J. (2007). Is parent involvement still important? Principal, 86(4), 8. 
Wikipedia (2010). Haiti. Retrieved February 21, 2010, from http://en.wikipedia.org/wiki/Haiti

Zephir, F. (2004). The Haitian Americans. Westport, Connecticut: Greenwood Press. 
APPENDIX A

\section{INTERVIEW PROTOCOL}

1. How long has your child been participating in a program for children with disabilities?

2. How do you see this child functioning in the home and are there differences to his/her functioning at school?

3. What can you tell me about the process that put your child in the ESE program?

4. How do you feel about this child and is different from the way you felt before he/she was placed?

5. How do you think being in this program has affected your child?

6. How has it affected you or how you feel about this child?

7. How do members of your family feel about this child?

8. What expectations do you have for this child in the future?

9. How are they different from the ones you have for your other children, if any, and why?

10. What are your greatest concerns about this child?

11. How do you see your child as an adult?

12. How do you get involved in this child's education?

13. What do you wish the teacher knew about your child that would help to better educate your child?

14. What is your greatest need as a parent of a child with a disability?

15. How can the school assist you in meeting your needs?

16. What barriers are preventing you from participating in your child's education?

17. Are there other comments you want to make? 


\section{APPENDIX B}

\section{CONSENT/ASSENT TO PARTICIPATE IN A RESEARCH STUDY}

Title: Analysis of Haitian Parents' Perceptions of the Education of Their Children with Disabilities

This form is to request your consent to interview you and to use information contained in your child's school records. We also request that you give your permission to attend any meetings held at your child's school during the course of this research study.

The investigator this research study is Josee Gregoire, a Miami-Dade County Public Schools employee and a graduate student at FIU. The study will analyze parental perceptions of their children with disabilities and attempt to determine what they perceive is their role in the educational process. It will also try to identify what needs Haitian parents of children with disabilities have and how the Miami-Dade public school system can meet those needs.

If you decide to participate in this study you will be asked to participate in an interview with follow-up sessions as needed during the course of data collection. You will be asked questions on the nature of your child's disability, the relationship between you and your child and the interactions you have with the school.

-You will be asked about your participation at parent meetings, conferences and any other communication you have with school personnel.

-You will also be asked to give permission to go through your child's school records to see if any information is available regarding contact with you.

The interviews will be approximately one hour in length unless you are not able for some reason to devote that time. The location for the interview will be of your choosing. We can break it up to fit into your schedule and follow-up sessions can be scheduled at your convenience.

There are no known risks to you as a participant in the study or in reviewing our child's records. If you feel that some of the questions asked during the interview are embarrassing or private to you, you may choose not to answer those questions. When participating in the interview, you can ask to take a break, if you get upset or feel uncomfortable. There may be no direct benefits to you for being in this study. However, this research will provide information on Haitian parents of children with disabilities attending Miami-Dade County Public Schools and their needs.

The research will also provide information to professionals working with Haitian children with disabilities to better serve the needs of the children and their parents. At the end of the study, we will write a report that will discuss what we found from interviewing the entire group of Haitian parents. All personal information collected during the course of the research study will be kept private. No Child or family will be identified individually. We will report the findings as a 
group with no identifying information listed. There is no cost to you for participating in this study.

No individually identifiable information will be reported. All data in this research is private and will not be shared with anyone not directly related to the study unless required by law. Only the responses will be presented in publications, reports and at conferences. You may ask questions about the study at any time. You can agree not to participate in this research study prior to the interview. If after the interview you change your mind, you may request that your information be removed from the study at any time and no one will be upset with you. Your child will continue to receive the same services from Miami-Dade County Public Schools whether you choose to participate in this research study or not.

If you would like to know more about this research, you can contact me, Ms. Josee Gregoire, at (305) 336-1383. If you feel that you were mistreated, or you have questions about being in the study you may contact Dr. Patricia Price, the Chairperson of the FIU Institutional Review Board at (305) 348-2618 or (305) 348-2494.

Please check the appropriate answer to indicate agreement or disagreement with being interviewed and to have your child's records reviewed during this research study.

I agree to be interviewed and to have my child's school records reviewed during the study.

I do not agree to being interviewed and to have my child's school records reviewed during the study.

If you have had all of your questions answered to your liking and you would like to be part of the study sign below.

Print Name of Student

Signature of Parent
Print Name of Parent

Date

I have explained the research procedure, subject rights and answered questions asked by the participant. I have offered him/her a copy of this informed consent form. 
JOSEE GREGOIRE

Born, Haiti

1988

B.A., Political Science

Florida International University

Miami, Florida

1995

M.S,

Exceptional Student Education

Florida International University

Miami, Florida

2010-2015

Florida Teachers' Certificate

Educational Leadership

Emotionally Handicapped

ESOL Endorsement

2007-Current

Assistant Principal

North Miami Elementary

Miami, Florida

2002-2007

Staffing Specialist K-12

Miami-Dade County Public Schools

Miami, Florida

1995-2002

Pre-k Staffing Specialist

Miami-Dade County Public Schools

Miami, Florida

$1988-1995$

Teacher

Pre-k Program for Children with

Disabilities

Miami-Dade County Public Schools

Miami, Florida

\section{PUBLICATIONS AND PRESENTATIONS}

Lucky, L. \& Gregoire, J, (1999). The effectiveness of early childhood education: How is it measured and by whom? The YAI National Institute for People with Disabilities, 20th Annual International Conference, New York City, N.Y. 
Gregoire, J. (1999), What every parent needs to know about the early years. The Mayor's children summit, Miami, Florida.

Gregoire, J. and Lucky, L.( 2002). Evaluating the effectiveness of a public school prekindergarten program for children with disabilities. COER 2002. Proceedings of Appreciating Scholarship. The first annual college of education research conference, Miami, Florida. 